\title{
Bioinformatics analysis and identification of hub genes and immune-related molecular mechanisms in chronic myeloid leukemia
}

\author{
Fangyi Yao ${ }^{1}$, Cui Zhao ${ }^{1}$, Fangmin Zhong ${ }^{1}$, Tingyu Qin ${ }^{1}$, Shuqi Li $^{1}$, Jing Liu ${ }^{1}$, Bo Huang ${ }^{1}$, Xiaozhong Wang ${ }^{\text {Corresp. } 1}$ \\ 1 Jiangxi Province Key Laboratory of Laboratory Medicine, Department of Clinical Laboratory, the Second Affiliated Hospital of Nanchang University, \\ Nanchang, Jiangxi, China \\ Corresponding Author: Xiaozhong Wang \\ Email address: wangxiaozhong@ncu.edu.cn
}

Background. Chronic myeloid leukemia $(\mathrm{CML})$ is a malignant hyperplastic tumor of the bone marrow originating from pluripotent hematopoietic stem cells. The advent of tyrosine kinase inhibitors (TKIs) has greatly improved the survival rate of patients with CML. However, TKI-resistance leads to the disease recurrence and progression. This study aimed to identify immune-related genes (IRGs) associated with CML progression.

Methods. We extracted the gene's expression profiles from the Gene Expression Omnibus (GEO). Bioinformatics analysis was used to determine the differentially expressed IRGs of CML and normal peripheral blood mononuclear cells (PBMCs). Functional enrichment and gene set enrichment analysis (GSEA) were used to explore its potential mechanism. Hub genes were identified using Molecular Complex Detection (MCODE) and the CytoHubba plugin. The hub genes' diagnostic value was evaluated using the receiver operating characteristic (ROC). The relative proportions of infiltrating immune cells in each CML sample were evaluated using CIBERSORT. Quantitative real-time PCR (RT-qPCR) was used to validate the hub gene expression in clinical samples.

Results. A total of 31 differentially expressed IRGs were identified. GO analyses revealed that the modules were typically enriched in the receptor ligand activity, cytokine activity, and endopeptidase activity. KEGG enrichment analysis of IRGs revealed that CML involved Th17 cell differentiation, the NFkappa B signaling pathway, and cytokine-cytokine receptor interaction. A total of 10 hub genes were selected using the PPI network. GSEA showed that these hub genes were related to the gammainterferon immune response, inflammatory response, and allograft rejection. ROC curve analysis suggested that six hub genes may be potential biomarkers for CML diagnosis. Further analysis indicated that immune cells were associated with the pathogenesis of CML. The RT-qPCR results showed that proteinase 3 (PRTN3), cathepsin G (CTSG), matrix metalloproteinase 9 (MMP9), resistin (RETN), eosinophil derived neurotoxin (RNase2), eosinophil cationic protein (ECP,RNase3) were significantly elevated in CML patients' PBMCs compared with healthy controls.

Conclusions. These results improved our understanding of the functional characteristics and immunerelated molecular mechanisms involved in CML progression and provided potential diagnostic biomarkers and therapeutic targets. 


\section{Bioinformatics analysis and identification of hub genes and} 2 immune-related molecular mechanisms in chronic myeloid 3 leukemia

4

5

6 7

8

Fangyi Yao ${ }^{1}$, Cui Zhao ${ }^{1}$, Fangmin Zhong ${ }^{1}$, Tingyu Qin ${ }^{1}$, Shuqi Li ${ }^{1}$, Jing Liu ${ }^{1}$, Bo Huang ${ }^{1}$, Xiaozhong Wang ${ }^{1}$

${ }^{1}$ Jiangxi Province Key Laboratory of Laboratory Medicine, Department of Clinical Laboratory, the Second Affiliated Hospital of Nanchang University, Nanchang, Jiangxi, China.

Corresponding Author:

Xiaozhong Wang ${ }^{1}$

Jiangxi Province Key Laboratory of Laboratory Medicine, Department of Clinical Laboratory, the Second Affiliated Hospital of Nanchang University, Nanchang, Jiangxi 330006, China. Email address: wangxiaozhong@ncu.edu.cn

\section{Abstract}

\section{Background}

Chronic myeloid leukemia (CML) is a malignant hyperplastic tumor of the bone marrow originating from pluripotent hematopoietic stem cells. The advent of tyrosine kinase inhibitors (TKIs) has greatly improved the survival rate of patients with CML. However, TKI-resistance leads to the disease recurrence and progression. This study aimed to identify immune-related genes (IRGs) associated with CML progression.

\section{Methods}

We extracted the gene's expression profiles from the Gene Expression Omnibus (GEO). Bioinformatics analysis was used to determine the differentially expressed IRGs of CML and normal peripheral blood mononuclear cells (PBMCs). Functional enrichment and gene set enrichment analysis (GSEA) were used to explore its potential mechanism. Hub genes were identified using Molecular Complex Detection (MCODE) and the CytoHubba plugin. The hub genes' diagnostic value was evaluated using the receiver operating characteristic (ROC). The relative proportions of infiltrating immune cells in each CML sample were evaluated using CIBERSORT. Quantitative real-time PCR (RT-qPCR) was used to validate the hub gene expression in clinical samples. 
35

36

37

38

39

40

41

42

43

44

45

46

47

48

49

50

51

52

53

54

55

56

57

58

59

60

61

62

63

64

65

\section{Results}

A total of 31 differentially expressed IRGs were identified. GO analyses revealed that the modules were typically enriched in the receptor ligand activity, cytokine activity, and endopeptidase activity. KEGG enrichment analysis of IRGs revealed that CML involved Th17 cell differentiation, the NF-kappa B signaling pathway, and cytokine-cytokine receptor interaction. A total of 10 hub genes were selected using the protein-protein interaction (PPI) network. GSEA showed that these hub genes were related to the gamma-interferon immune response, inflammatory response, and allograft rejection. ROC curve analysis suggested that six hub genes may be potential biomarkers for CML diagnosis. Further analysis indicated that immune cells were associated with the pathogenesis of CML. The RT-qPCR results showed that proteinase 3 (PRTN3), cathepsin G (CTSG), matrix metalloproteinase 9 (MMP9), resistin (RETN), eosinophil derived neurotoxin (RNase2), eosinophil cationic protein (ECP, RNase3) were significantly elevated in CML patients' PBMCs compared with healthy controls.

\section{Conclusions}

These results improved our understanding of the functional characteristics and immune-related molecular mechanisms involved in CML progression and provided potential diagnostic biomarkers and therapeutic targets.

\section{Introduction}

54 Chronic myeloid leukemia (CML) is a malignant, clonal, and proliferative disease originating from hematopoietic stem cells (Nash 1999). CML is characterized by the presence of a BCRABL1 fusion gene. This disease is classified into a chronic phase (CP), accelerated phase (AP), and a blast phase (BP). Tyrosine kinase inhibitors (TKIs), especially Imatinib (IM), have been used to treat CML with favorable outcomes (Lugo et al. 1990). However, TKI use may lead to side effects and drug resistance. This in turn may decrease TKI efficacy in some patients (Hehlmann 2012). Thus, it is important to understand the mechanisms underlying CML and determine potential biomarkers.

2 Studies have proposed that gene expression-based characteristics may be used in CML diagnosis and prognosis (Vinhas et al. 2017), but they have not been made a part of routine clinical care due to insufficient validation cohorts. A number of new biomarkers have emerged following the validation of large-scale gene expression datasets (Feng et al. 2020). The 
66

67

68

69

70

71

72

73

74

75

76

77

78

79

80

81

82

83

84

85

\section{Materials \& Methods}

\section{Data collection}

88

89

90

91

92

93

94

95

\section{Identifying IRGs}

establishment of multiple gene expression profiles makes it possible to identify reliable CML biomarkers. New evidence has suggested that the immune system, consisting of immune-related genes (IRGs) and tumor-infiltrating immune cells, is vital during cancer initiation and progression (Zou \& Hu 2020). A number of recent studies have constructed prognostic immune signals for use in the diagnosis and prognosis of a variety of cancers (Geng et al. 2021). Hu et al. (2021) constructed a seven-IRG signature using data from the Gene Expression Omnibus (GEO) database to predict glioblastoma multiform prognosis. This signature also revealed the potential functions of these genes in glioblastoma occurrence and development (Hu et al. 2021). Zhu et al. (2020) predicted the survival rate of patients with acute myeloid leukemia with a normal karyotype by constructing a six-IRG signature to determine their prognostic characteristics. However, the clinical role of IRGs in CML has yet to be determined.

In this study, we identified IRGs using the data from the GEO database and performed network analyses to assess the molecular mechanisms underlying CML progression. Further, the biological processes involved were analyzed using gene ontology (GO) enrichment and kyoto encyclopedia of genes and genomes (KEGG) pathways for the differentially expressed genes. Moreover, the top $10 \mathrm{hub}$ genes screened via protein-protein interaction (PPI) network were selected for their functional similarity, and their diagnostic value was assessed. Our results established the first comprehensive network of IRGs and provided a useful framework for explaining CML's molecular mechanisms at the systems biology level.

The gene expression dataset GSE100026 ( $\mathrm{Li}$ et al. 2020) was downloaded from the GEO database (GPL18573 Illumina NextSeq 500) and annotated using R software equipped with annotation files. This dataset included five CML chronic-phase (CML-CP) peripheral blood mononuclear cell (PBMCs) samples, five CML blast-period (CML-BP) PBMCs samples, and five normal PBMCs samples. We acquired 2,498 genes from the ImmPort database (https://immport.niaid.nih.gov) (Bhattacharya et al. 2018). Hallmark gene sets were extracted from the MSigDB database (http://software.broadinstitute.org/gsea/msigdb/genesets.jsp). The overview of the workflow is shown in Fig. 1. 
97 We converted the RNA sequencing (RNA-seq) data from RNA-Seq by Expectation 98 Maximization (RSEM) into transcripts per kilobase million (TPM) expression profiles. Data 99 analysis was performed using the limma package in R (Ritchie et al. 2015). The cutoffs were set 100 as $|\log \mathrm{FC}|>1, P<0.05$, and the false discovery rate (FDR) $<0.05$ to screen for differentially 101 expressed genes. The intersection of the differentially expressed genes and the immune genes 102 was used to find the IRGs. These results were displayed on a volcano map and a heat map (Xue 103 et al. 2020).

\section{PPI network construction and module analysis}

105 We used the Metascape database (https://metascape.org/gp/index.html\#/main/step1) to perform 106 GO and KEGG pathway enrichment analyses. We identified the most enriched biological 107 pathways and functions related to the IRGs (Zhou et al. 2019). We then constructed a protein108 protein interaction (PPI) network of IRGs using the Search Tool for the Retrieval of Interacting 109 Genes (STRING) online database (http://string-db.org, version 10). The network was visualized 110 in Cytoscape (version 3.6.1) software (Szklarczyk et al. 2017). We used the Molecular Complex 111 Detection (MCODE) and cytoHubba plug-ins to identify the merged network's hub genes. These 112 genes serve as diagnostic markers (Chin et al. 2014).

\section{Verifying diagnostic markers}

114 We performed receiver operating characteristic (ROC) curve analysis on each screened 115 diagnostic marker in the GSE100026 dataset to verify its accuracy. R's pROC package was used

116 for ROC curve analysis. Regarding the interpretation of AUC results, a test with an area $>0.9$ 117 indicates high accuracy, 0.7-0.9 as moderate accuracy, 0.5-0.7 as low accuracy, and 0.5 as a 118 chance result (Akobeng 2007). Genes with an AUC value greater than 0.7 were retained.

\section{Gene set enrichment analysis (GSEA)}

120 GSEA is a statistical method used to determine whether genes from a particular pathway or other 121 predefined gene sets are differentially expressed in different phenotypes. Reactome pathways 122 were analyzed with GSEA using clusterProfiler to define each functional cluster. 123 C2.all.v6.2.symbols.gmt was selected as the reference gene set. A false discovery rate $<0.1$ and $124 P$-value $<0.01$ were set as the cut-off criteria.

\section{Estimating immune cell type fractions}

126 CIBERSORT is a bioinformatics method used to evaluate the composition of immune cells 127 through standardized gene expression transformation (Chen et al. 2018). The CIBERSORT 
128 algorithm (CIBERSORT R script v1.03; http://cibersort.stanford.edu/) was used to analyze the 129 immune infiltration of the CML-CP-Normal, CML-BP-Normal, and CML-CP-CML-BP datasets, 130 respectively. This analysis helped us assess differences in immune cell infiltration among CML131 CP, CML-BP, and healthy samples.

132 Analysis of the relationship between hub genes and immune cells

133 The relationship between the six hub genes and the infiltrating immune cells was analyzed in 134 three datasets. Hub genes expression levels were divided into low- or high-expression groups 135 based on the genes' median expression levels. The Wilcoxon rank-sum test was used to analyze 136 the difference of immune cell infiltration between the high and low levels of hub genes. Box 137 plots were used to visualize the difference in infiltrating immune cell expression between both 138 groups.

\section{Sample collection}

140 Peripheral blood samples were collected from patients in the Second Affiliated Hospital of 141 Nanchang University from March 2020 to February 2021. CML patients were pathologically 142 diagnosed and categorized into CML-CP $(n=25)$ and CML-BP $(n=25)$ groups. The normal 143 samples $(n=25)$ were obtained from healthy individuals. The characteristics of CML patients and 144 healthy donors are presented in Table 1. PBMCs were isolated by density gradient centrifugation 145 on a Ficoll-Paque (Sigma, USA) according to the manufacturer's protocol. Our study was 146 approved by the Ethics Committee of the Second Affiliated Hospital of Nanchang University 147 (approval no. 2017096) and all aspects of the study followed the guidelines set forth by the 148 Helsinki Declaration. All participants provided signed informed consent.

149 RNA isolation and quantitative real-time PCR (RT-qPCR)

150 Total RNA was extracted using TRIzol reagent (Invitrogen). Reverse transcription was 151 performed using a PrimeScript RT Reagent Kit (TaKaRa, Dalian, China). We performed RT152 qPCR using SYBR Premix Ex Taq (TaKaRa) on a ABI 7500 Real-Time PCR System (Applied 153 Biosystems, Foster City, CA, USA). All primers were designed and synthesized by Nanjing 154 Genscript Biotech Co., Ltd. GAPDH was used as the internal reference. The primers used in RT155 qPCR assay are listed in Table 2. Indicated genes' fold changes were calculated using the $2^{-\Delta \Delta \mathrm{Ct}}$ 156 method.

157 Statistical analysis 
158 Statistical analysis was performed with $\mathrm{R}$ Software (version 3.6.2). A $P$-value $<0.05$ was 159 considered to be statistically significant.

160

\section{Results}

162

163

164

165

166

167

168

169

170

171

172

173

174

175

176

177

178

179

180

181

182

183

184

185

186

187

188

\section{Identification of IRGs}

We used the limma package to analyze the differences between the CML-CP-Normal (CML$\mathrm{CP}=5$, Normal=5), CML-BP-Normal (CML-BP=5, Normal=5), and CML-CP-CML-BP (CML$\mathrm{CP}=5, \mathrm{CML}-\mathrm{BP}=5$ ) datasets. We ascertained where the differential genes and IRGs in each dataset intersected. From the three datasets, we identified 336, 159, and 376 differential genes, respectively (Fig. 2G, 2H, 2I). CML-CP-Normal's clustering heat map is shown in Fig. 2A and its volcano map is shown in Fig. 2D. CML-BP-Normal's clustering heat map is shown in Fig. 2B and its volcano map is shown in Fig. 2E. CML-CP-CML-BP's clustering heat map is shown in Fig. 2C and its volcano map is shown in Fig. 2F. Thirty-one IRGs were obtained from the intersection of the differential genes and the immune genes in the three datasets (Fig. 2J).

\section{Functional enrichment analysis of IRGs}

Thirty-one IRGs were enriched and analyzed using the Metascape database. The top GO terms related to biological processes (BC) were neutrophil activation, neutrophil degranulation, and neutrophil activation involved in immune response (Fig. 3A). The top GO terms related to cellular components (CC) included secretory granule lumen, cytoplasmic vesicle lumen, and vesicle lumen. The top GO terms related to molecular functions (MF) included receptor ligand activity, cytokine activity, and endopeptidase activity. KEGG analysis indicated that these IRGs were mainly enriched in Th17 cell differentiation, NF-kappa B signaling pathway, and cytokinecytokine receptor interaction (Fig. 3B).

\section{PPI network analysis of IRGs}

STRING was used to generate the PPI network, and the Cytoscape tool was used to visualize interactions between 31 IRGs (Fig. 4A and 4B). The cytoHubba and MCODE plug-ins were used to analyze the hub genes with maximum correlation criterion (MCC), and genes with the top 10 scores were identified as hub genes (Fig. 4C and 4D). Among these genes, AZU1, CAMP, CCL5, CTSG, MMP9, MPO, PRTN3, RETN, RNASE2, and RNASE3 showed the highest node scores, suggesting that they may play causative roles in CML progression.

GSEA analysis of hub genes 
189 GSEA were performed on the 10 hub genes obtained from the GSE10002 dataset. The median of 190 each gene expression value was used to categorize the patients into high or low groups. The 191 msigDB database's hallmark gene set was used as a reference in our analysis. We identified 192 different significant pathways between the higher- and lower-gene-expression groups. $P<0.05$ 193 and $|\mathrm{NES}|>1.0$ was considered to be significant. As shown in Fig. 5, most pathways enriched in 194 the hub genes high-expression cohort were immune- related, including interferon gamma 195 response, IL2-STAT5 signaling pathway, complement, allograft rejection.

196 Hub genes for CML diagnosis

197 To explore the accuracy of the top 10 hub genes as the diagnostic biomarkers for CML, the ROC 198 curves were plotted, respectively (Fig. 6). Six hub genes with an AUC value greater than 0.7 199 were used as diagnostic markers. Notably, the AUC for CTSG reached 0.96, and was the largest 200 among the 10 hub genes. The other AUC values were 0.82, 0.74, 0.88, 0.84, and 0.88 for MMP9 201 , PRTN3, RETN, RNASE2, and RNASE3, respectively. These results suggest that the 6 hub genes 202 may serve as diagnostic biomarkers for CML.

203 Immune infiltration analysis

204 We performed immune infiltration analysis on three datasets (Figs. 7-9). The distribution of the 20522 types of infiltrating immune cells in each sample showed there to be immunological 206 differences between the two groups (Fig. 7A, 8A, 9A). The abundance of immune cells in each 207 sample is illustrated as a heatmap in Fig. 7B, 8B, 9B; the correlation between each of the 208 infiltrated immune cell types is shown in Fig. 7C, 8C, 9C; and the violin plot in Fig. 7D, 8D, 9D 209 visualizes the differences in each type of immune cell between the 2 groups.

210 Relationships between the hub genes and immune cells

211 We analyzed the relationship between hub genes CTSG, MMP9, PRTN3, RETN, RNASE2 and 212 RNASE3 and immune cells in the three datasets, respectively (Figs. 10-12). In the CML-BP213 Normal datasets, CTSG, MMP9, PRTN3, RNASE2 and RNASE3 were significantly associated 214 with the infiltration of monocytes, mast cells resting and NK cells (Fig. 10). In the CML-CP215 CML-BP datasets, the six hub genes were significantly associated with the infiltration of $\mathrm{CD} 8^{+} \mathrm{T}$ 216 cells, $\mathrm{CD}^{+}{ }^{\mathrm{T}} \mathrm{T}$ cells, NK cells resting, macrophages M0, eosinophils (Fig. 12). The results suggest 217 that there is some correlation between the six hub genes and immune response in CML patients.

218 Validating hub genes expression in clinical samples 
219 To further assess the CTSG, MMP9, PRTN3, RETN, RNASE2, and RNASE3 expression levels in 220 CML, we perform RT-qPCR to search the mRNA expression of the hub genes in PBMCs of the 221 healthy individuals and CML-CP/BP patients (Fig. 13). The results showed that the expression of 222 CTSG, MMP9, PRTN3, RETN, RNASE2, and RNASE3 were dramatically upregulated in CML223 CP comparing to the normal cells $(P<0.001)$. Compared with CML-CP samples, the expression 224 of CTSG, RETN, RNASE2, and RNASE3 were significantly lower in the CML-BP samples. The 225 expression trend was consistent with the GSE100026 dataset.

226 In the present study, the correlation between clinical features of CML and the expression 227 levels of hub genes were analyzed. As shown in Table 3, the expression of CTSG was positively 228 associated with white blood cell (WBC) number $(r=0.4827, P=0.0007)$ and haemoglobin $229(r=0.3135, P=0.0241)$. The expression of PRTN3 was positively associated with haemoglobin $230(r=0.3326, P=0.0128)$. The expression of $R E T N$ was positively associated with WBC number $231(r=0.3832, P=0.0027)$. Furthermore, the expression levels of $M M P 9, R N A S E 2$, and $R N A S E 3$ in 232 PBMCs from patients with CML did not correlate with WBC number, haemoglobin, and platelet 233 (Table 3).

234

\section{Discussion}

236 Approximately $15 \%$ of all new leukemia cases are CML according to American Cancer Society 237 estimates (Apperley 2015). The use of TKIs such as imatinib and nilotinib significantly 238 improved the efficacy of CML treatment. TKI treatment over 12 months led $66 \%$ of CML 239 patients to have a complete cytogenetic response. Major molecular biological responses were 240 noted in $40 \%$ of patients and the 10 -year overall survival rate reached $85 \%-90 \%$ (Melo \& Barnes 241 2007). However, drug resistance has emerged with diversified and individual characteristics, 242 seriously affecting TKI efficacy (Deininger et al. 2000). Therefore, it is important to develop 243 therapeutic targets aside from ABL kinase.

244 Recent immunotherapy research has brought to light the relationship between tumors and 245 immunity. The positive response to immunotherapy typically depends on the interaction between 246 tumor cells and immune regulation. Previous studies have investigated the role of immunity in 247 various cancers and have found that immune effector cells such as T and NK cells can effectively 248 eliminate tumor cells (Sconocchia et al. 2005). However, immune cells also confer resistance 249 when the oncogenic signaling pathway is activated or the protective mechanism against cell 
250 death is active (Held et al. 2016). These findings are ready to be applied clinically. For example, 251 the National Institute for Health and Care Excellence (NICE) recommends that some patients 252 using National Healthcare System (NHS) adopt new CAR-T treatments for lymphoma (Mahase 253 2021). We focused on immune molecule and immune cell changes during CML progression and 254 attempted to locate IRGs to extract key genes and explore effective diagnostic markers. We 255 identified 31 IRGs by comparing the transcriptional expression profiles of CML-CP, CML-BP, 256 257 258 259 260 261 262 263 264 265 266 267 268 269 and healthy samples. GO and KEGG enrichment analysis revealed that these enriched modules and pathways are closely related to immune cell activation and the immune response observed in CML. The top 10 hub genes associated with CML were identified in the PPI network. Of these ten, six hub genes (CTSG, MMP9, PRTN3, RETN, RNASE2 and RNASE3) may be used as CML diagnostic markers. Their expressions were significantly associated with a variety of immune cell disorders by immune infiltration analysis.

We performed GO enrichment analysis to investigate the 31 IRGs' BPs. Of the MF annotations, receptor ligand activity, cytokine activity, endopeptidase activity were found to be significantly associated with CML occurrence and development. We performed KEGG analysis to determine the biological functions of the 31 immune-related genes associated with CML. Th17 cell differentiation, NF-kappa B signaling pathway, and cytokine-cytokine receptor interaction were the three most significantly enriched pathways. Interestingly, some enriched pathways were associated with CML. For instance, NF- $\mathrm{KB}$ is activated through the canonical IKK pathway and plays distinct roles in the pathogenesis of myeloid and lymphoid leukemias induced by BCR-ABL1. This confirms that NF- $\mathrm{KB}$ and IKKs are targets for $\mathrm{Ph}(+)$ leukemias 272 (ANK) with a potent major histocompatibility complex that had unrestricted cytotoxic activity 273 and suppressed malignant hematopoiesis. These observations support the use of 274 autologous ANK therapy to prevent the relapse of CML after autologous marrow transplantation 275 or the use of ANK to purge CML marrow for autologous transplantation (Cervantes et al. 1996). 276 Autology-activated NK cells can inhibit primitive CML progenitors in long term cultures. CML 277 cells can avoid immune escape in vivo, resulting in NK cell-mediated immune destruction 278 (Cervantes et al. 1996). IFN- $\gamma$ released by activated T or NK cells modulate the sensitivity of 279 CML cells to TKI, thereby interfering with the therapeutic effect of TKI on CML. This effect 
280 suggests that inflammation-mediated BCR-ABL1 independent resistance mechanisms are 281 significant (Held et al. 2016).

282 In the PPI network identified in the present study, 10 IRGs were determined to be the most 283 significant hub genes, with multiple interactions in the network. The 10 hub genes' AUCs were 284 analyzed with regard to diagnostic value. An AUC value greater than 0.7 was the screening 285 criteria. Six hub genes (CTSG, MMP9, PRTN3, RETN, RNASE2, RNASE3) were determined to 286 be diagnostic markers for CML. The AUC values ranged from 0.74-0.96. The RT-qPCR results 287 showed that CTSG, MMP9, PRTN3, RETN, RNASE2, RNASE3 were significantly elevated in 288 PBMCs of CML patients compared with healthy controls. GSEA showed that these hub genes 289 were predominantly associated with the interferon gamma response, inflammatory response, and 290 allograft rejection.

291 Cathepsin G (CTSG) plays an important role in host defense and can escape intracellular 292 monitoring systems by preventing degradation of foreign proteins. CTSG is broadly expressed in 293 acute myeloid leukemia (AML) blasts and leukemia stem cells (Alatrash 2013). Recent studies 294 have shown that CTSG is an effective target for the immunotherapy of AML and acute lymphoid 295 leukemia (ALL) (Groborz et al. 2019). We found that CTSG was highly-expressed in CML-CP 296 and CML-BP. We also found that the AUC of CTSG was 0.96, indicating that it has high 297 diagnostic value. Matrix metalloproteinase 9 (MMP9) upregulation in leukemic cells promotes 298 the blast crisis in CML (Nakahara et al. 2014). In chronic lymphocytic leukemia (CLL), MMP9 299 contributes to CLL pathology by regulating cell survival and migration, promoting angiogenesis, 300 and is associated with poor prognosis (Aguilera-Montilla et al. 2019). It has been shown that the 301 ID1 transcriptional inhibitor-MMP9 axis can enhance the invasiveness of BCR-ABL1 302 transformed leukemia cells (Nieborowska-Skorska et al. 2006). Human proteinase 3 (PRTN3) is 303 a leukemia-associated antigen. The identified PRTN3(235)-epitope can be used to study the role 304 of CD4+Th- and Treg-cells in immune responses against PRTN3 in leukemia patients (Berlin et 305 al. 2015). Resistin (RETN), a proinflammatory cytokine, is elevated in a number of pathological 306 disorders, including leukemia. The serum RETN level in all patients was elevated and may serve 307 as a potential clinical diagnostic marker to detect the recurrence of leukemia (El-Baz et al. 2013). 308 Human eosinophil derived neurotoxin (EDN, RNase2) and eosinophil cationic protein 309 (ECP, RNase3) sequences may have up to a 92\% identity in their promoter regions (Wang et al. 310 2009). It produces host defense effects by promoting leukocyte activation, maturation, and 
311 chemotaxis. Niini et al. (2002) demonstrated that RNase2 was abnormally expressed in

312 childhood acute lymphoblastic leukemia (a cDNA array) and was associated with prognosis.

313 These findings are consistent with our work, which suggests that there may be a close 314 relationship between CML and immune composition.

315 Our study had some limitations. First, the sample size was small; a larger sample size is 316 needed to verify our results. Second, some clinical data are lacking due to the heterogeneity, long 317 duration, and difficulty of follow-up of CML. It is difficult for us to assess the relationship 318 between risk indicators and patient stratification based on the severity of CML. In order to 319 determine the value of hub genes related to CML as diagnostic and therapeutic biomarkers, more 320 clinical variables should be used for further external verification. Third, the potential mechanism 321 of the six hub genes in CML progression is still not well understood and additional studies may 322 be needed to determine the six hub genes' underlying molecular mechanisms in CML.

323

\section{Conclusions}

325 Ours is the first study to comprehensively identify IRGs in CML. In total, 31 IRGs and 10 hub genes were identified and may serve as novel potential targets for the diagnosis and treatment of CML. However, more molecular experiments are needed to validate our findings.

\section{References}

330

331

332

333

334

335

336

Aguilera-Montilla N, Bailón E, Ugarte-Berzal E, Uceda-Castro R, Prieto-Solano M, GarcíaMartínez E, Samaniego R, Van den Steen P, Opdenakker G, García-Marco J, and GarcíaPardo A. 2019. Matrix metalloproteinase-9 induces a pro-angiogenic profile in chronic lymphocytic leukemia cells. Biochemical and biophysical research communications 520:198-204. 10.1016/j.bbrc.2019.09.127

Akobeng AK. 2007. Understanding diagnostic tests 3: Receiver operating characteristic curves. Acta Paediatr 96:644-647. 10.1111/j.1651-2227.2006.00178.x

Alatrash G. 2013. Targeting cathepsin G in myeloid leukemia. Oncoimmunology 2:e23442. 10.4161/onci. 23442

Apperley JF. 2015. Chronic myeloid leukaemia. Lancet 385:1447-1459. 10.1016/s01406736(13)62120-0

Berlin C, Kowalewski D, Schuster H, Mirza N, Walz S, Handel M, Schmid-Horch B, Salih H, Kanz L, Rammensee H, Stevanović S, and Stickel J. 2015. Mapping the HLA ligandome landscape of acute myeloid leukemia: a targeted approach toward peptide-based immunotherapy. Leukemia 29:647-659. 10.1038/leu.2014.233

Bhattacharya S, Dunn P, Thomas C, Smith B, Schaefer H, Chen J, Hu Z, Zalocusky K, Shankar R, Shen-Orr S, Thomson E, Wiser J, and Butte A. 2018. ImmPort, toward repurposing of 
open access immunological assay data for translational and clinical research. Scientific data 5:180015. 10.1038/sdata.2018.15

Cervantes F, Pierson B, McGlave P, Verfaillie C, and Miller J. 1996. Autologous activated natural killer cells suppress primitive chronic myelogenous leukemia progenitors in longterm culture. Blood 87:2476-2485.

Chen B, Khodadoust M, Liu C, Newman A, and Alizadeh A. 2018. Profiling Tumor Infiltrating Immune Cells with CIBERSORT. Methods in molecular biology (Clifton, NJ) 1711:243259. 10.1007/978-1-4939-7493-1_12

Chin C, Chen S, Wu H, Ho C, Ko M, and Lin C. 2014. cytoHubba: identifying hub objects and sub-networks from complex interactome. BMC systems biology:S11. 10.1186/1752-05098-s4-s11

Deininger MW, Goldman JM, and Melo JV. 2000. The molecular biology of chronic myeloid leukemia. Blood 96:3343-3356.

El-Baz HA, Mosa TE, Elabd EM, Ramadan A, Elharoun AS, Elmorsy EA, and Fouda MI. 2013. Serum adiponectin and resistin levels in de novo and relapsed acute lymphoblastic leukemia children patients. Iran J Public Health 42:504-510.

Feng XQ, Nie SM, Huang JX, Li TL, Zhou JJ, Wang W, Zhuang LK, and Meng FJ. 2020. Circular RNA circHIPK3 serves as a prognostic marker to promote chronic myeloid leukemia progression. Neoplasma 67:171-177. 10.4149/neo_2018_181129N908

Geng Q, Shen Z, Li L, and Zhao J. 2021. COL1A1 is a prognostic biomarker and correlated with immune infiltrates in lung cancer. PeerJ 9:e11145. 10.7717/peerj.11145

Groborz K, Kołt S, Kasperkiewicz P, and Drag M. 2019. Internally quenched fluorogenic substrates with unnatural amino acids for cathepsin $\mathrm{G}$ investigation. Biochimie 166:103111. 10.1016/j.biochi.2019.05.013

Hehlmann R. 2012. How I treat CML blast crisis. Blood 120:737-747. 10.1182/blood-2012-03380147

Held S, Heine A, Kesper A, Schönberg K, Beckers A, Wolf D, and Brossart P. 2016. Interferon gamma modulates sensitivity of CML cells to tyrosine kinase inhibitors. Oncoimmunology 5:e1065368. 10.1080/2162402x.2015.1065368

Hsieh M, and Van Etten R. 2014. IKK-dependent activation of NF- $\kappa B$ contributes to myeloid and lymphoid leukemogenesis by BCR-ABL1. Blood 123:2401-2411. 10.1182/blood2014-01-547943

Hu L, Han Z, Cheng X, Wang S, Feng Y, and Lin Z. 2021. Expression Profile Analysis Identifies a Novel Seven Immune-Related Gene Signature to Improve Prognosis Prediction of Glioblastoma. Front Genet 12:638458. 10.3389/fgene.2021.638458

Li SQ, Liu J, Zhang J, Wang XL, Chen D, Wang Y, Xu YM, Huang B, Lin J, Li J, and Wang XZ. 2020. Transcriptome profiling reveals the high incidence of hnRNPA1 exon 8 inclusion in chronic myeloid leukemia. $J$ Adv Res 24:301-310. 10.1016/j.jare.2020.04.016

Lugo TG, Pendergast AM, Muller AJ, and Witte ON. 1990. Tyrosine kinase activity and transformation potency of bcr-abl oncogene products. Science 247:1079-1082. 10.1126/science. 2408149

Mahase E. 2021. NICE recommends new CAR-T treatment for lymphoma for some patients on NHS. BMJ (Clinical research ed) 372:n184. 10.1136/bmj.n184

Melo JV, and Barnes DJ. 2007. Chronic myeloid leukaemia as a model of disease evolution in human cancer. Nat Rev Cancer 7:441-453. 10.1038/nrc2147 
392

393

394

395

396

397

398

399

400

401

402

403

404

405

406

407

408

409

410

411

412

413

414

415

416

417

418

419

420

421

422

423

424

425

426

427

428

Nakahara F, Kitaura J, Uchida T, Nishida C, Togami K, Inoue D, Matsukawa T, Kagiyama Y, Enomoto Y, Kawabata K, Chen-Yi L, Komeno Y, Izawa K, Oki T, Nagae G, Harada Y, Harada H, Otsu M, Aburatani H, Heissig B, Hattori K, and Kitamura T. 2014. Hes1 promotes blast crisis in chronic myelogenous leukemia through MMP-9 upregulation in leukemic cells. Blood 123:3932-3942. 10.1182/blood-2013-01-476747

Nash I. 1999. Chronic myeloid leukemia. N Engl J Med 341:765. 10.1056/nejm199909023411016

Nieborowska-Skorska M, Hoser G, Rink L, Malecki M, Kossev P, Wasik M, and Skorski T. 2006. Id1 transcription inhibitor-matrix metalloproteinase 9 axis enhances invasiveness of the breakpoint cluster region/abelson tyrosine kinase-transformed leukemia cells. Cancer research 66:4108-4116. 10.1158/0008-5472.Can-05-1584

Ritchie M, Phipson B, Wu D, Hu Y, Law C, Shi W, and Smyth G. 2015. limma powers differential expression analyses for RNA-sequencing and microarray studies. Nucleic acids research 43:e47. 10.1093/nar/gkv007

Sconocchia G, Lau M, Provenzano M, Rezvani K, Wongsena W, Fujiwara H, Hensel N, Melenhorst J, Li J, Ferrone S, and Barrett A. 2005. The antileukemia effect of HLAmatched NK and NK-T cells in chronic myelogenous leukemia involves NKG2D-targetcell interactions. Blood 106:3666-3672. 10.1182/blood-2005-02-0479

Szklarczyk D, Morris J, Cook H, Kuhn M, Wyder S, Simonovic M, Santos A, Doncheva N, Roth A, Bork P, Jensen L, and von Mering C. 2017. The STRING database in 2017: qualitycontrolled protein-protein association networks, made broadly accessible. Nucleic acids research 45:D362-D368. 10.1093/nar/gkw937

Vinhas R, Cordeiro M, Pedrosa P, Fernandes AR, and Baptista PV. 2017. Current trends in molecular diagnostics of chronic myeloid leukemia. Leuk Lymphoma 58:1791-1804. 10.1080/10428194.2016.1265116

Wang H, Ho P, Lan C, and Chang M. 2009. Transcriptional regulation of human eosinophil RNase 2 by the liver-enriched hepatocyte nuclear factor 4. Journal of cellular biochemistry 106:317-326. 10.1002/jcb.22008

Xue F, Yang L, Dai B, Xue H, Zhang L, Ge R, and Sun Y. 2020. Bioinformatics profiling identifies seven immune-related risk signatures for hepatocellular carcinoma. PeerJ 8:e8301. 10.7717/peerj.8301

Zhou Y, Zhou B, Pache L, Chang M, Khodabakhshi A, Tanaseichuk O, Benner C, and Chanda S. 2019. Metascape provides a biologist-oriented resource for the analysis of systems-level datasets. Nature communications 10:1523. 10.1038/s41467-019-09234-6

Zou Y, and Hu C. 2020. A 14 immune-related gene signature predicts clinical outcomes of kidney renal clear cell carcinoma. PeerJ 8:e10183. 10.7717/peerj.10183

PeerJ reviewing PDF | (2021:04:60516:2:0:NEW 21 Oct 2021) 
Table $\mathbf{1}$ (on next page)

Clinical characteristics 


\begin{tabular}{|l|c|c|c|}
\hline \multicolumn{4}{|c}{ Table 1. Clinical characteristics } \\
\hline Characteristic & CML-CP $(\mathrm{n}=25)$ & CML-BP $(\mathrm{n}=25)$ & Normal $(\mathrm{n}=25)$ \\
\hline $\begin{array}{l}\text { Age (years), median } \\
\text { (range) }\end{array}$ & $49(19-69)$ & $55(30-73)$ & $52(21-70)$ \\
\hline Male/female $(\mathrm{n} / \mathrm{n})$ & $13 / 12$ & $15 / 10$ & $14 / 11$ \\
\hline $\begin{array}{l}\text { WBC count, } \times 10^{9} / \mathrm{L}, \\
\text { median }(\text { range })\end{array}$ & $138.3(14.8-440.5)$ & $72.6(2.5-381.9)$ & $6.4(4.8-9.7)$ \\
\hline $\begin{array}{l}\text { Haemoglobin level } \\
(\mathrm{g} / \mathrm{L})\end{array}$ & $102(50-143)$ & $79(43-135)$ & $139(120-156)$ \\
\hline $\begin{array}{l}\text { Platelet count, } \times 10^{9} / \mathrm{L}, \\
\text { median (range) }\end{array}$ & $395(6-1309)$ & $50(10-183)$ & $223(105-318)$ \\
\hline
\end{tabular}

1 BP, blast phase; CML, chronic myeloid leukemia; CP, chronic phase; WBC, white blood cells. 2 
Table 2 (on next page)

RT-qPCR primers 
Table 2. RT-qPCR primers

\begin{tabular}{|l|l|}
\hline Primers & \multicolumn{1}{|c|}{ Sequences (5'-3') } \\
\hline GAPDH & Forward: ATGGTGAAGGTCGGTGTGAA \\
\hline & Reverse: GAGTGGAGTCATACTGGAAC \\
\hline CTSG & Forward: GAGTCAGACGGAATCGAAACG \\
\hline & Reverse: CGGAGTGTATCTGTTCCCCTC \\
\hline MMP9 & Forward: CGCAGACATCGTCATCCAGT \\
\hline PRTN3 & Reverse: CGCAGACATCGTCATCCAGT \\
\hline & Forward: CCCTGATCCACCCGAGATTC \\
\hline RETN & Reverse: GGTTCTCCTCGGGGTTGTAA \\
\hline & Forward: GTGTGCCGGATTTGGTTAGC \\
\hline RNASE2 & Reverse: GAGGGAACCAAGAGACCCAC \\
\hline & Forward: ATCAACGACGAGACCCTCCA \\
\hline RNASE3 & Reverse: AGGAGCTTGGCAGATGAGTG \\
\hline & Forward: GATCCACGGGATTCTCCACG \\
\hline & Reverse: GGAGCTTGGCAGATGAGTGA \\
\hline
\end{tabular}

1 


\section{Table 3 (on next page)}

Correlation of the expression of 6 hub genes with clinical characteristics of CML 
1

\begin{tabular}{|c|c|c|c|c|c|c|c|c|c|c|c|c|}
\hline Age & 0.0328 & 0.8476 & 0.0215 & 0.8041 & 0.0755 & 0.6429 & 0.0952 & 0.5738 & 0.0415 & 0.7928 & 0.0617 & 0.6952 \\
\hline Haemoglobin & 0.3135 & 0.0241 & 0.0952 & 0.6673 & 0.3326 & 0.0128 & 0.1884 & 0.5246 & 0.1268 & 0.4572 & 0.1668 & 0.3058 \\
\hline Platelet & 0.1769 & 0.2745 & 0.1364 & 0.4115 & 0.2328 & 0.1494 & 0.2159 & 0.1776 & 0.1675 & 0.3894 & 0.0364 & 0.8215 \\
\hline
\end{tabular}


Figure 1

Flow chart of methodologies applied in the study 


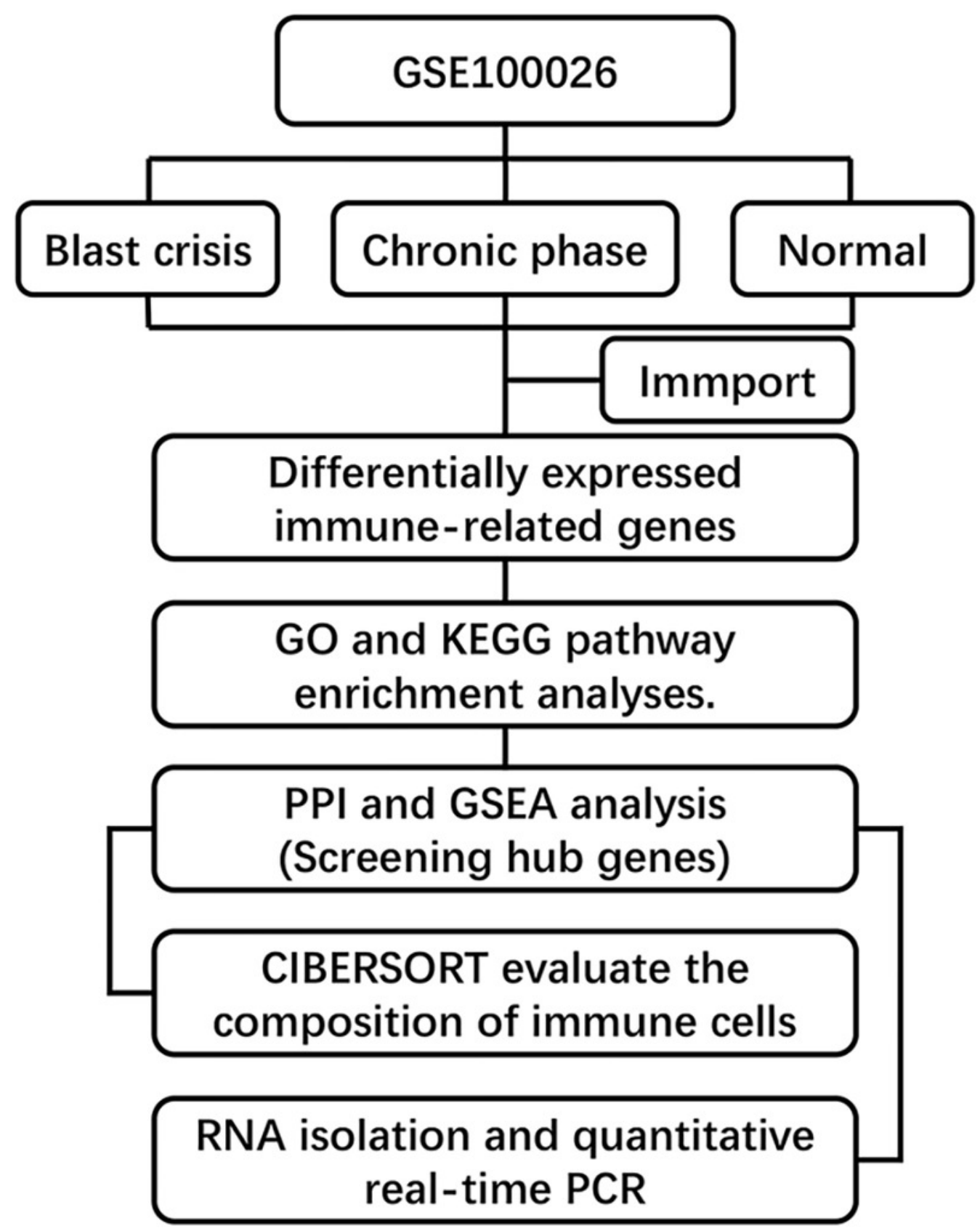




\section{Figure 2}

Identification of IRGs in CML

(A)-(C) Difference Analysis between CML-CP-Normal,CML-BP-Normal and CML-CP-CML-BP

Cluster Heat Map. (D)-(F) Difference Analysis between CML-CP-Normal, CML-BP-Normal and CML-CP-CML-BP Volcano Map. (G)-(I) CML-CP-Normal, CML-BP-Normal, CML-CP-CML-BP and Immune Gene Intersection Venn diagram. (J) Intersection venn diagram of the IRGs signifificantly associated with CML which were short-listed for the cross-validation.

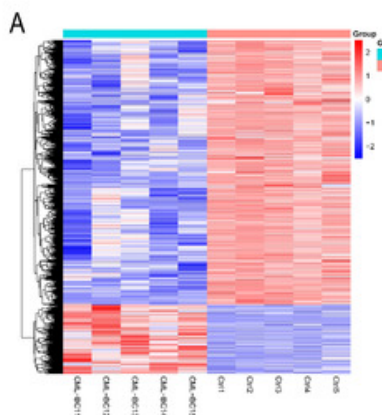

D

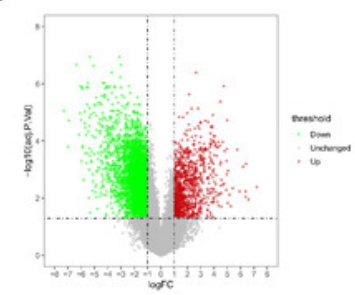

G

$\mathrm{H}$
B

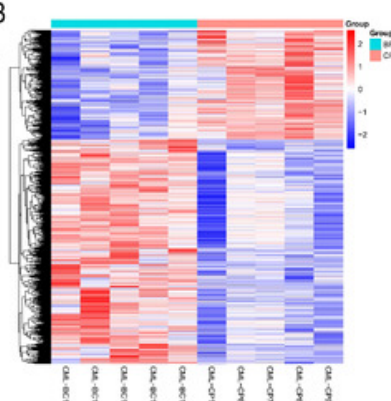

E

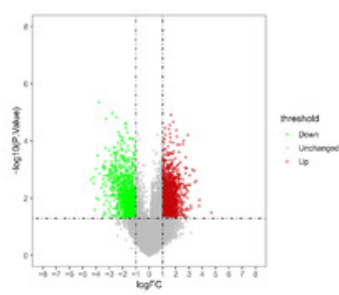

I

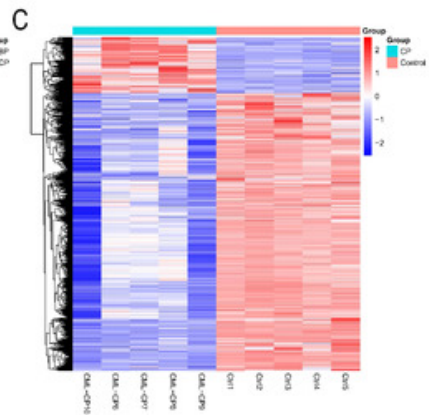

F
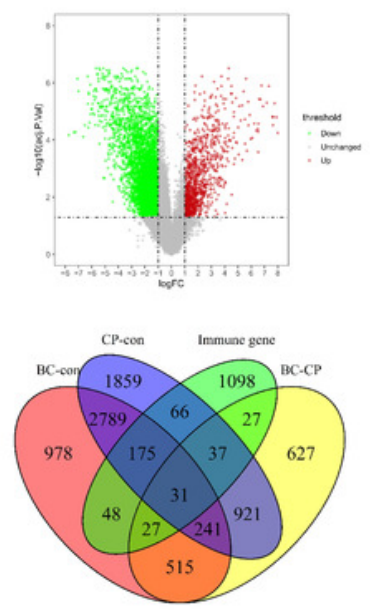
Figure 3

\section{GO term and KEGG pathway analysis for 31 IRGs}

(A) GO enrichment analysis. (B) KEGG enrichment analysis.

A

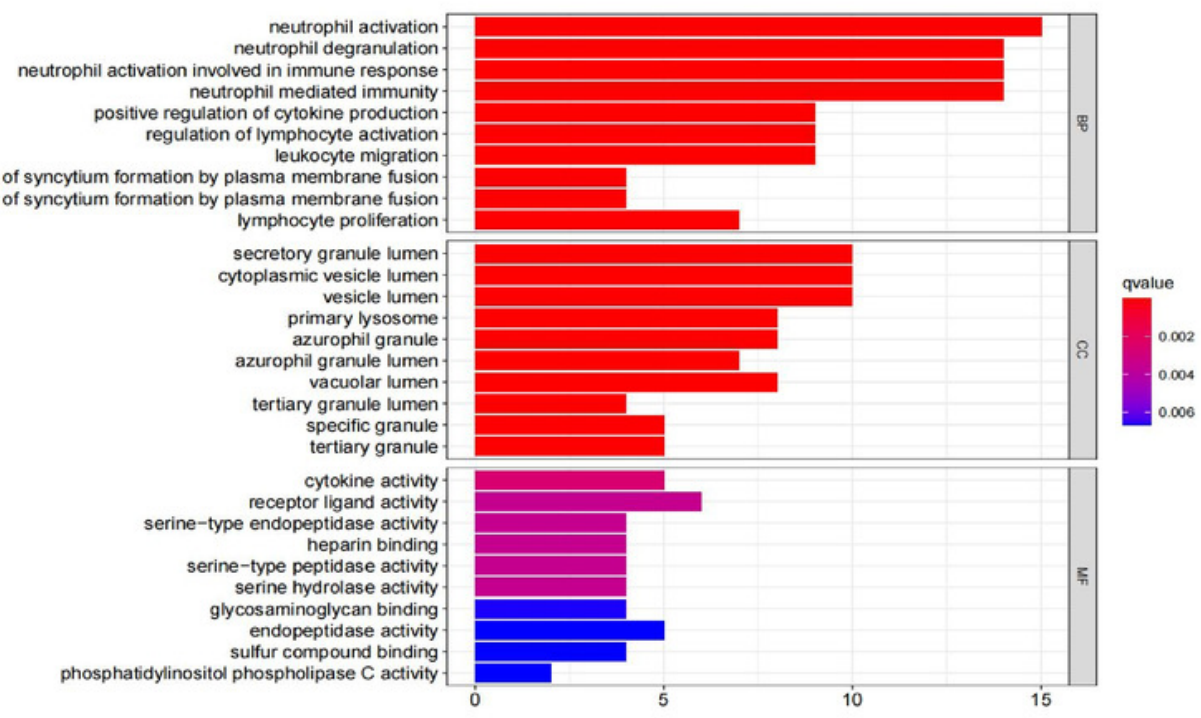

B

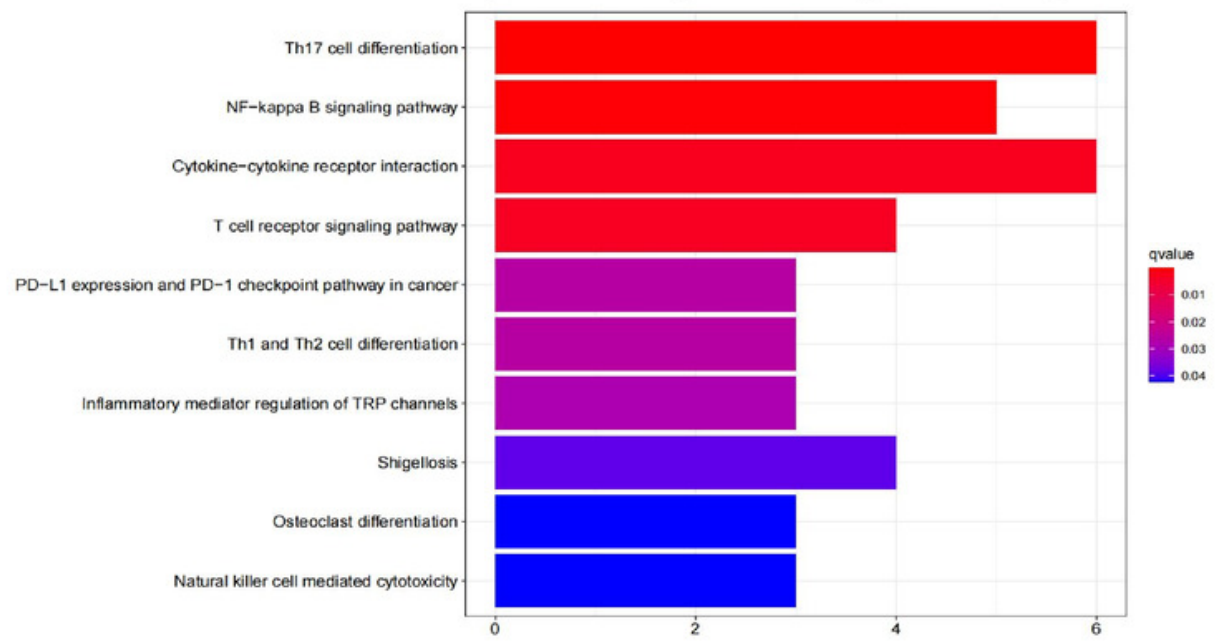


Figure 4

Significant modular analysis based on PPI network

(A)-(B) PPI network was constructed using a total of 31 IRGs. (C) 10 hub genes recognized by cytoHubba plug-in. (D) 10 hub gene recognized by MCODE plug-in.

A

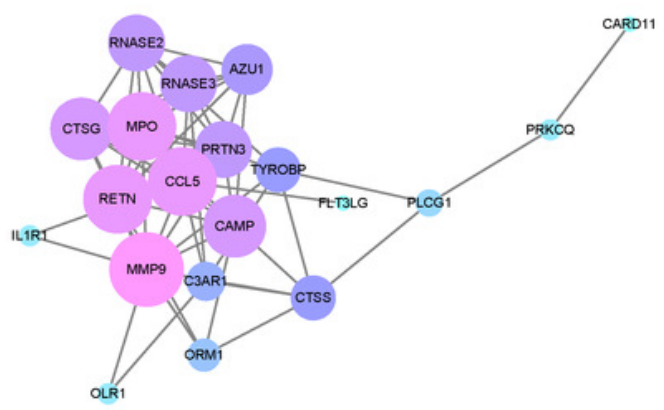

C

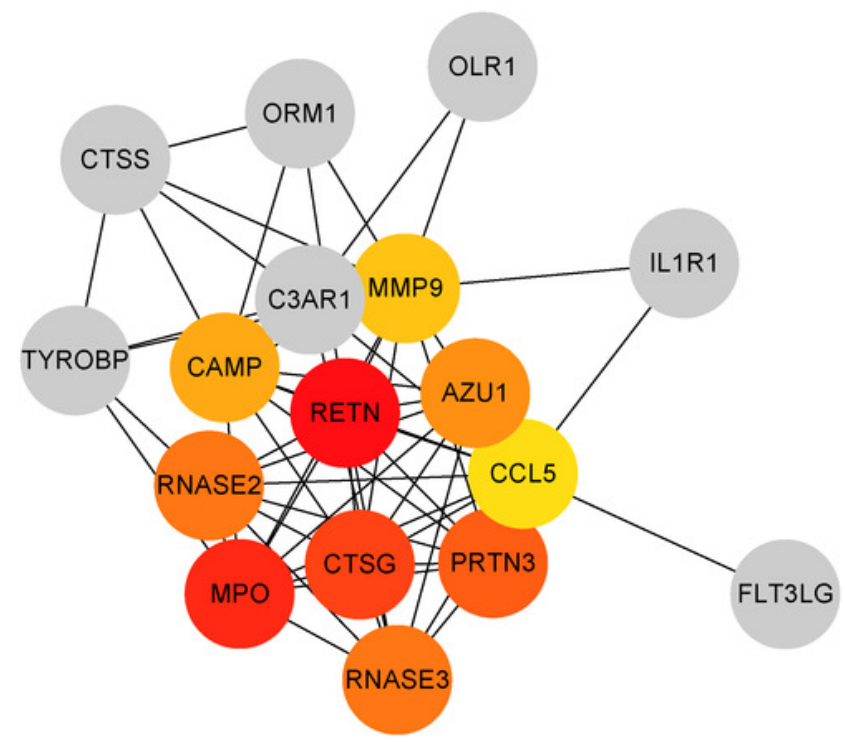

B

D
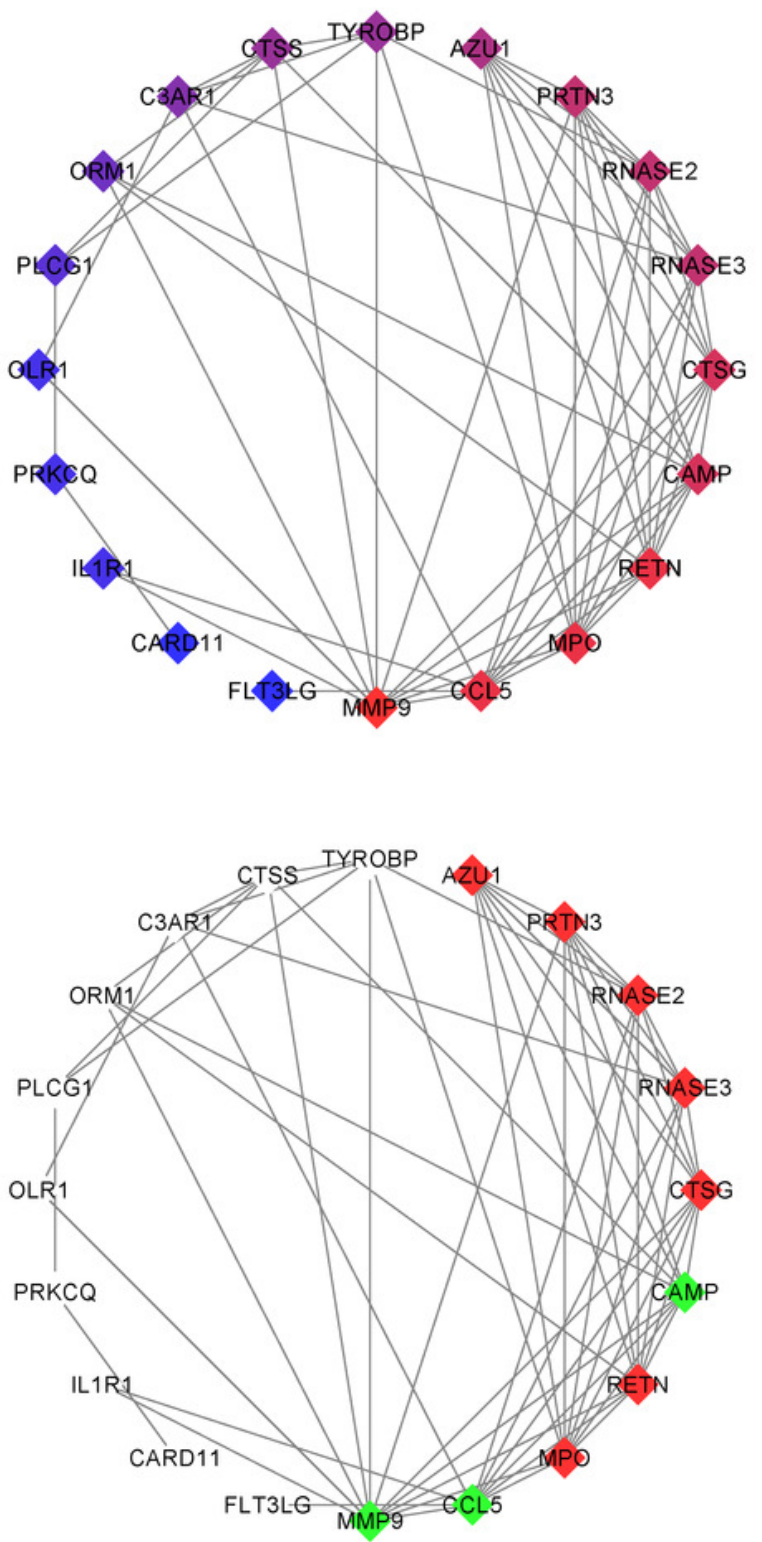
Figure 5

GSEA of the 10 hub gene expression profiles of the hallmark gene set

(A) AZU1. (B) CAMP. (C) CCL5. (D) CTSG. (E) MMP9. (F) MPO. (G) PRTN3. (H) RETN. (I) RNASE2. (J) RNASE3. 
A

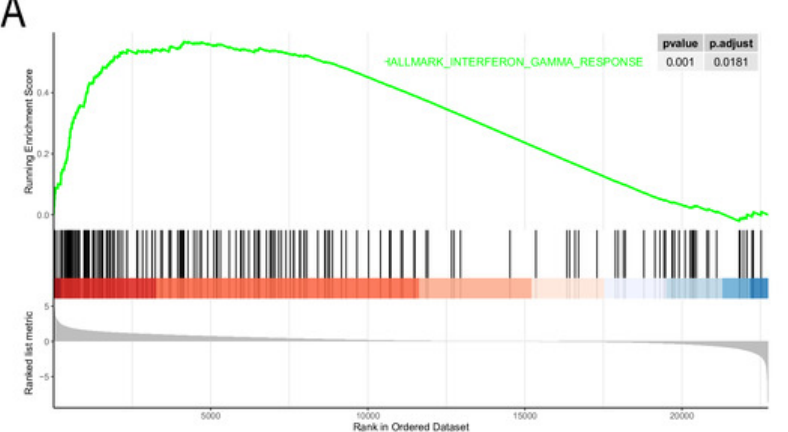

C

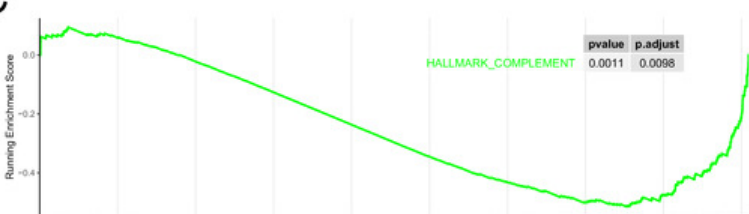

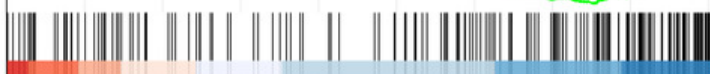

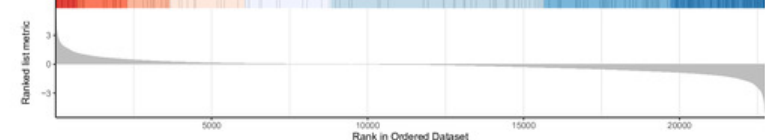

E

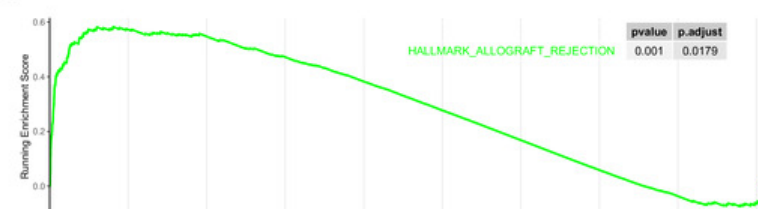

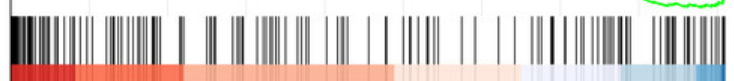

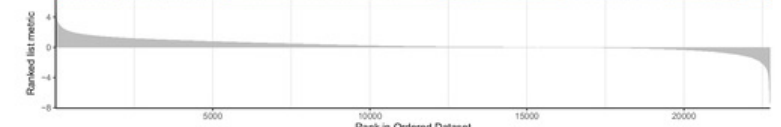

$$
\text { G }
$$

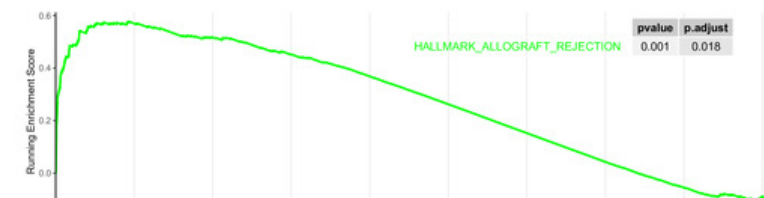

| ||||||||||||||||||||||||||||||||||||||||||||||||||||||||||||||||||

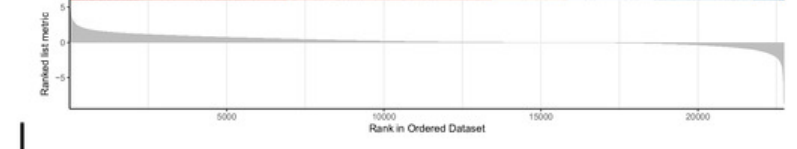

I

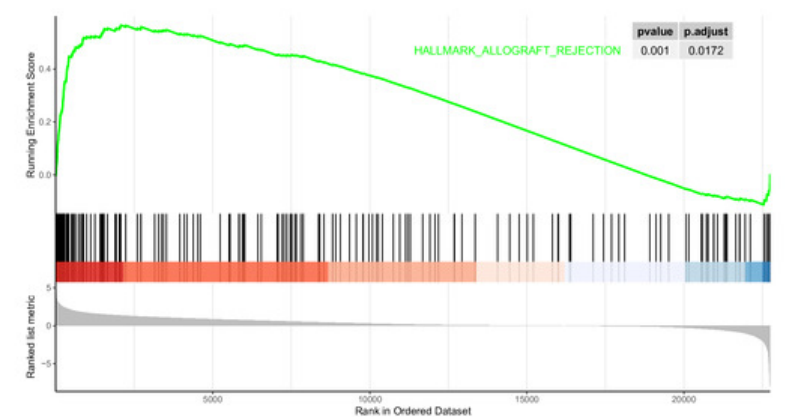

B

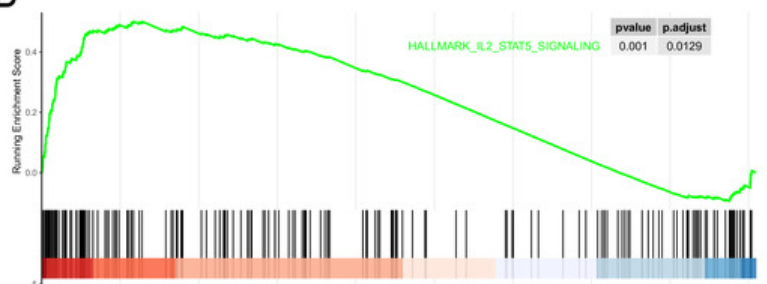

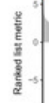

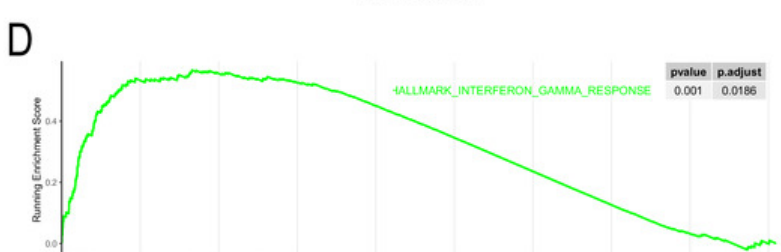

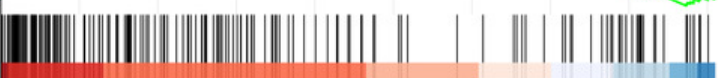

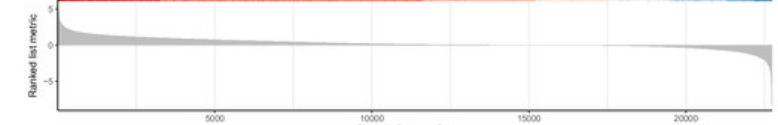

$\mathrm{F}$
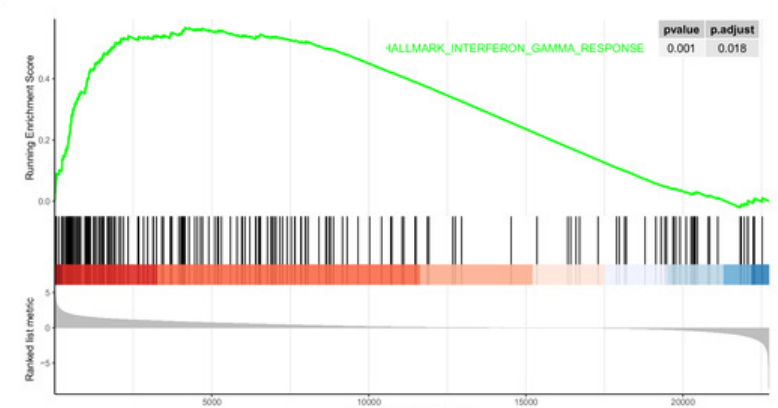

$\mathrm{H}$
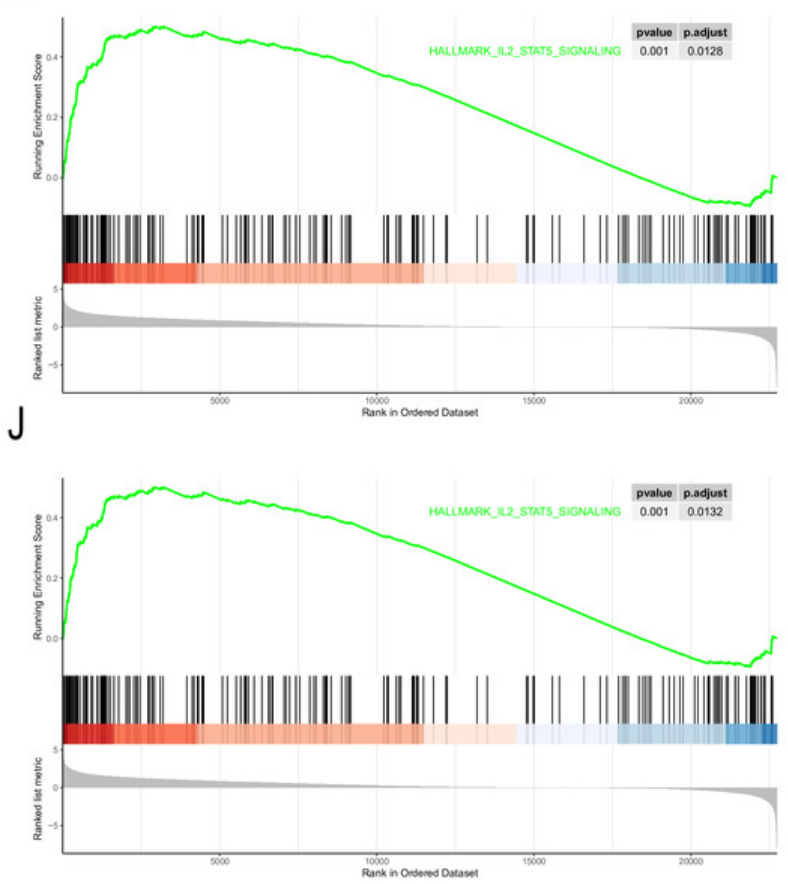
Figure 6

ROC curves were generated to validate the ability of diagnostic value of the 10 hub genes for CML

(A) AZU1. (B) CAMP. (C) CCL5. (D) CTSG. (E) MMP9. (F) MPO. (G) PRTN3. (H) RETN. (I)

RNASE2. (J) RNASE3. 

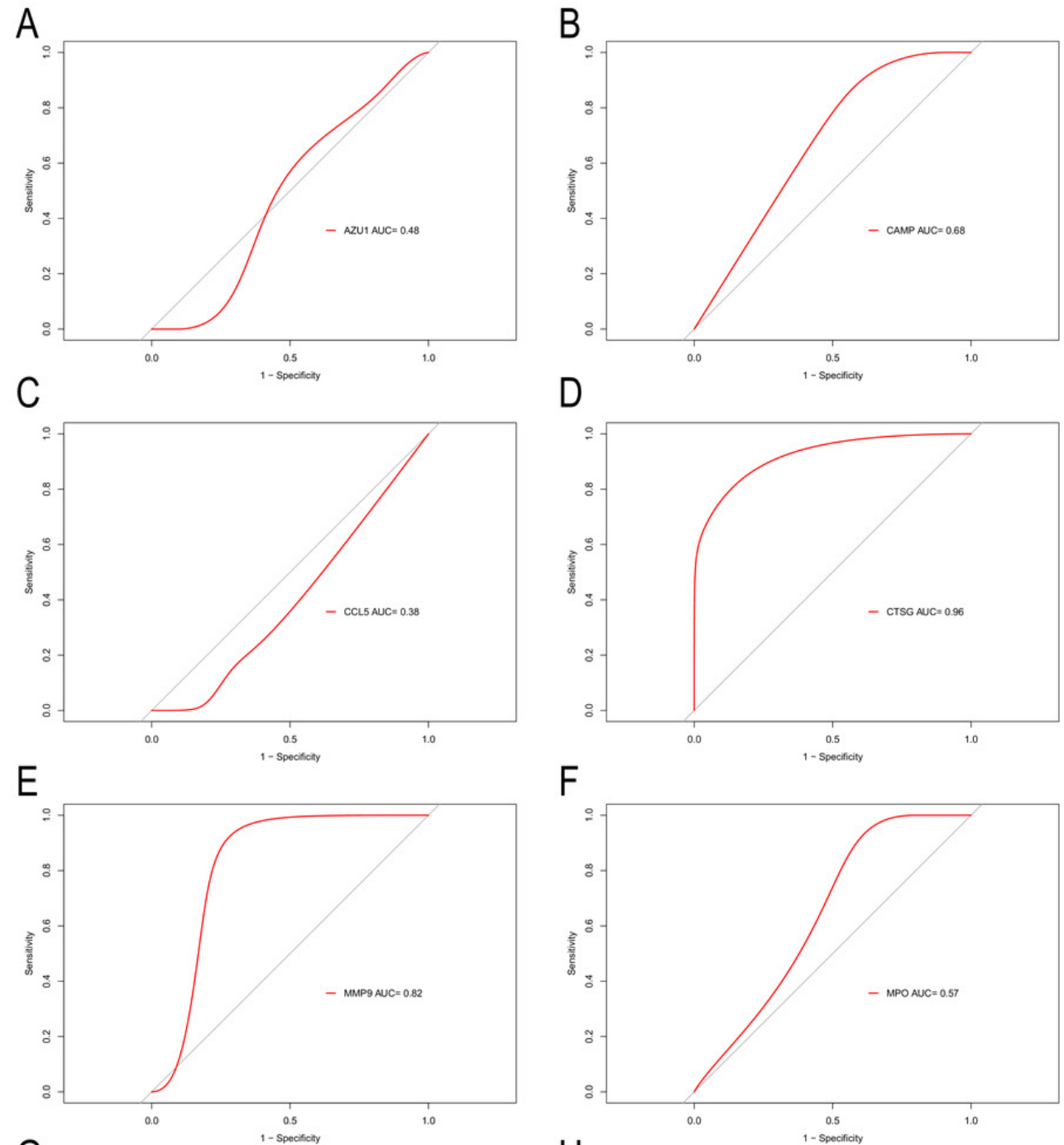

G

$\mathrm{H}$
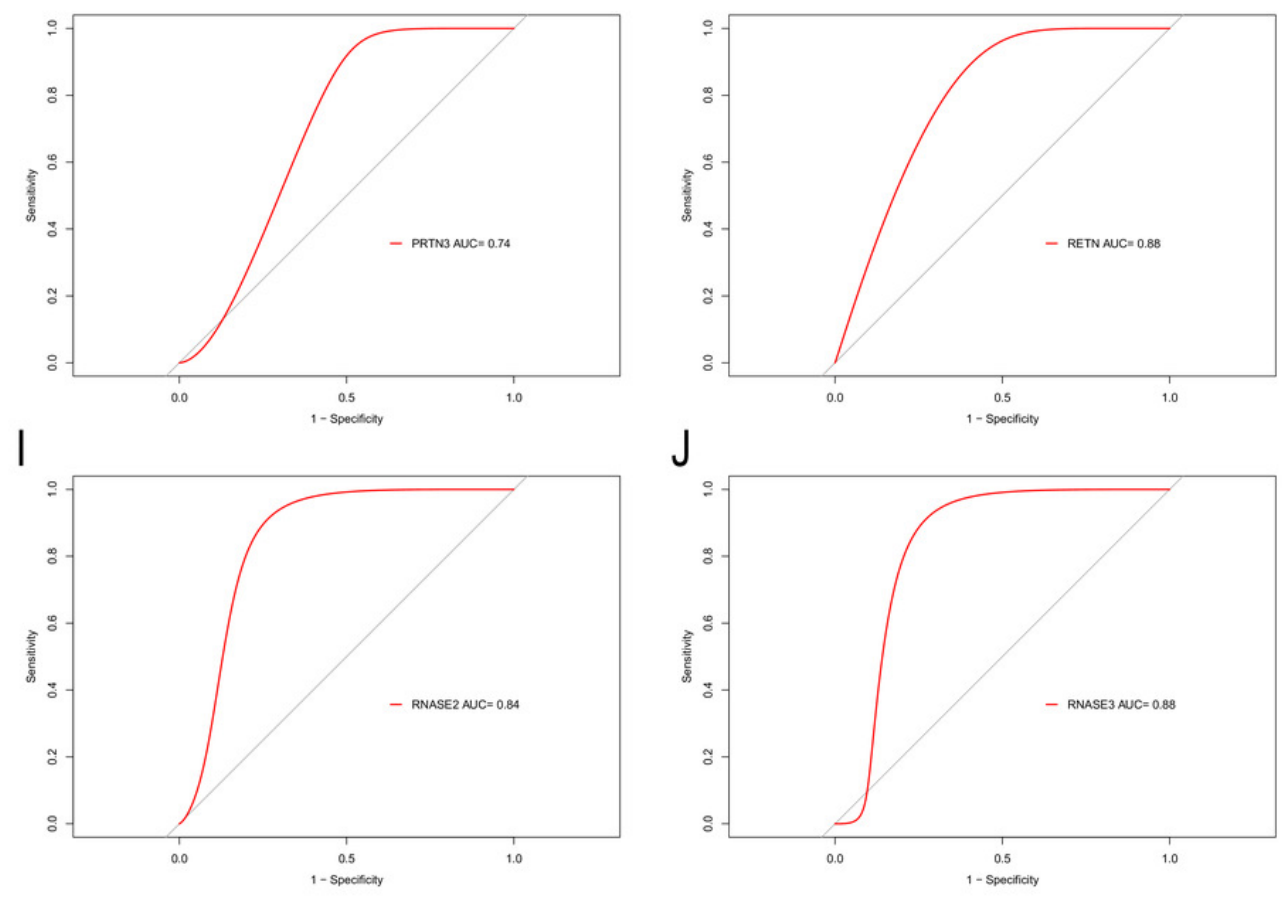

PeerJ reviewing PDF | (2021:04:60516:2:0:NEW 21 Oct 2021) 


\section{Figure 7}

Immune infiltration analysis of CML-CP and Normal

(A) 22 Immune cell composition of each sample. (B) Heat map of the 22 immune cell proportions in CML-CP and Normal. (C) The co-expression patterns among fractions of immune cells. (D) The violin graph shows the difference of immune infiltration between CML$\mathrm{CP}$ and Normal.

A

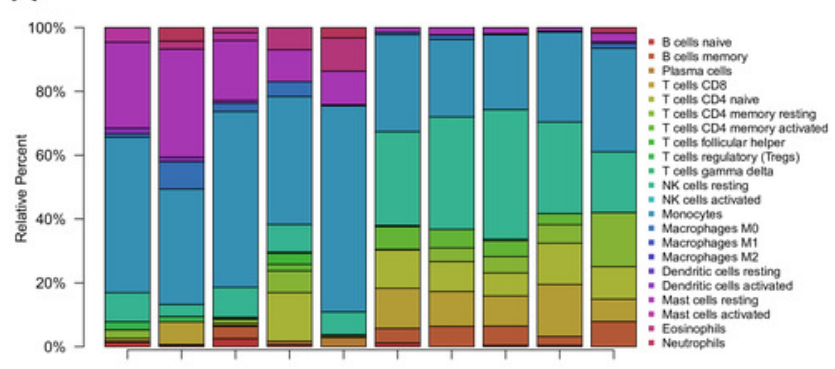

C

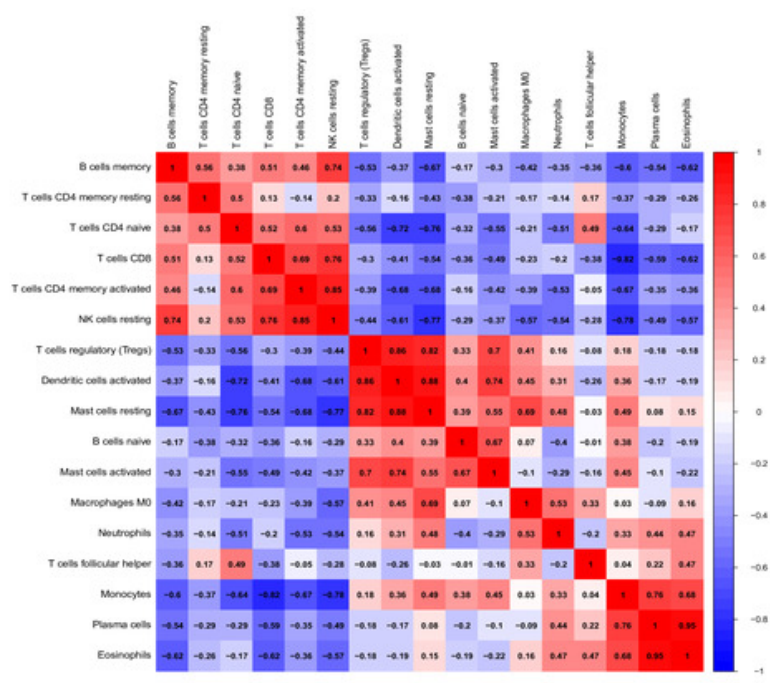

B

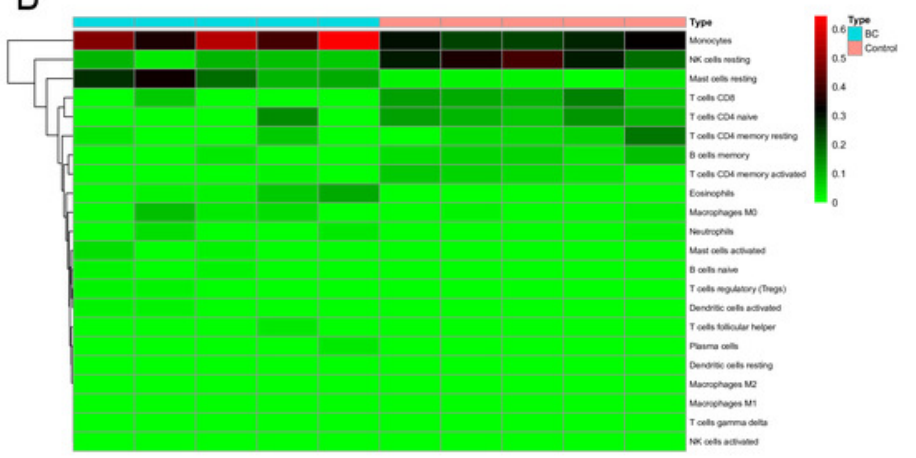

D

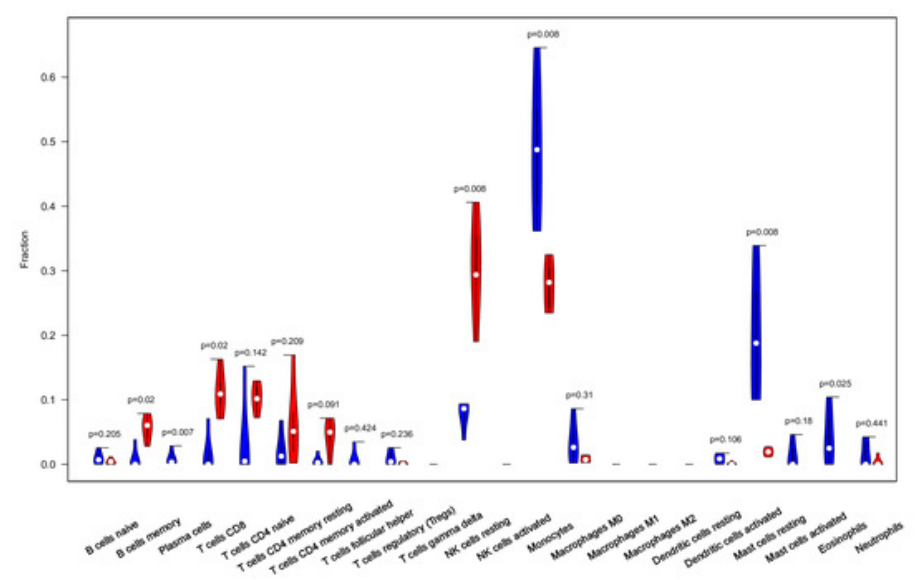




\section{Figure 8}

Immune infiltration analysis of CML-BP and Normal

(A) Immune cell composition of each sample. (B) Heat map of the 22 immune cell proportions in CML-BP and Normal. (C) The co-expression patterns among fractions of immune cells. (D) The violin graph shows the difference of immune infiltration between CML-BP and Normal.

A

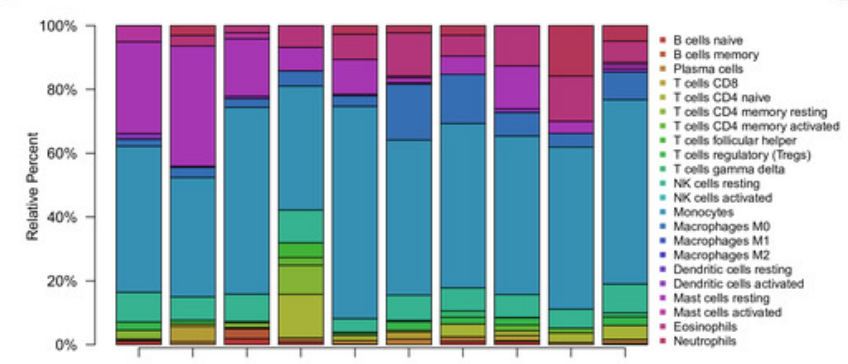

C

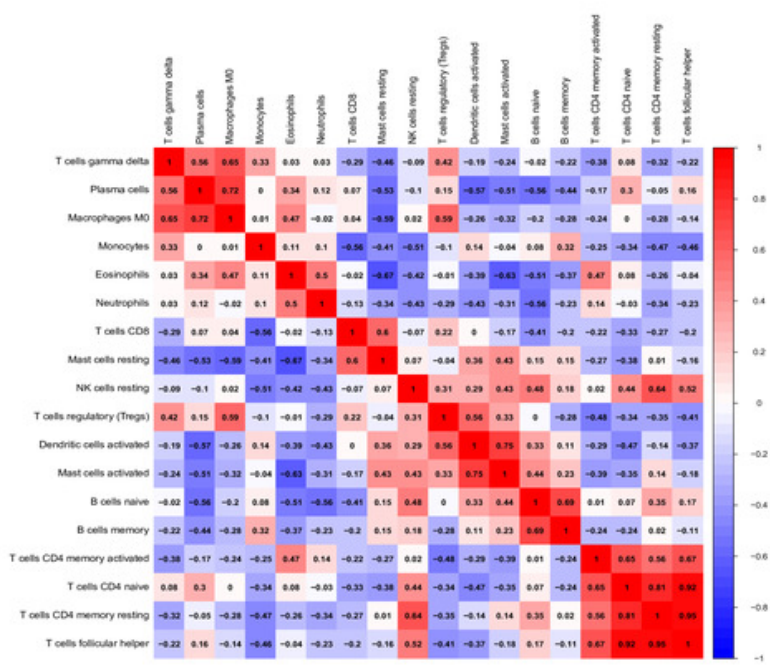

B

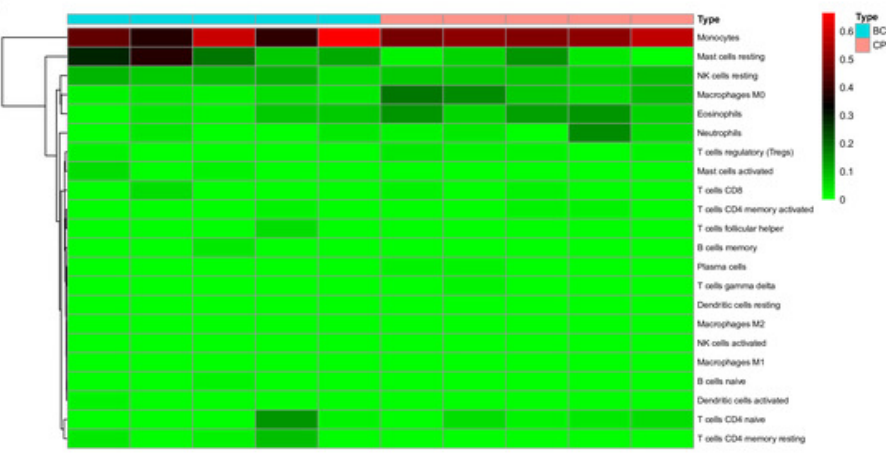

D

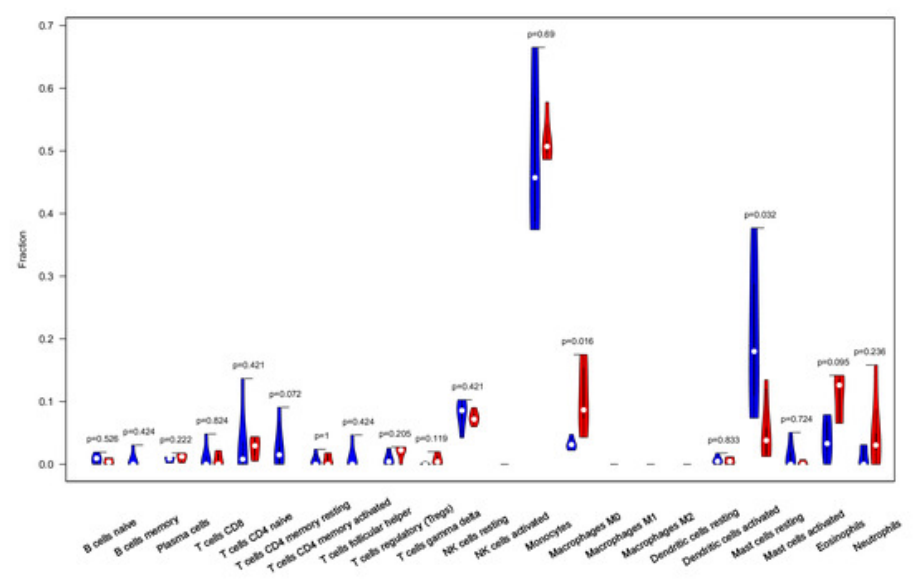




\section{Figure 9}

Immune infiltration analysis of CML-CP and CML-BP

(A) Immune cell composition of each sample. (B) Heat map of the 22 immune cell proportions in CML-CP and CML-BP. (C) The co-expression patterns among fractions of immune cells. (D) The violin graph shows the difference of immune infiltration between CML-CP and CML-BP.

A

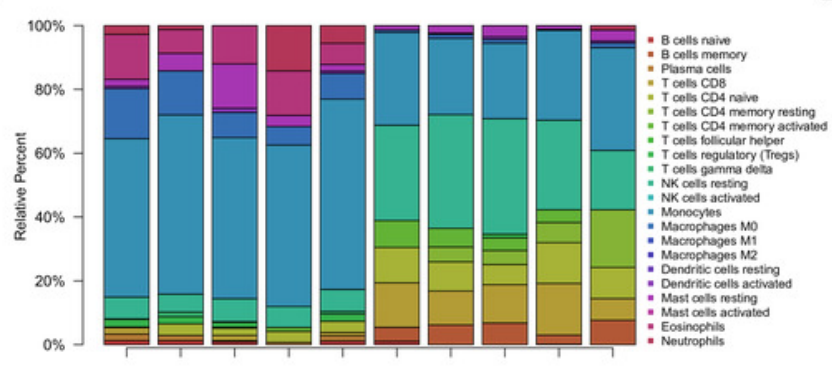

C

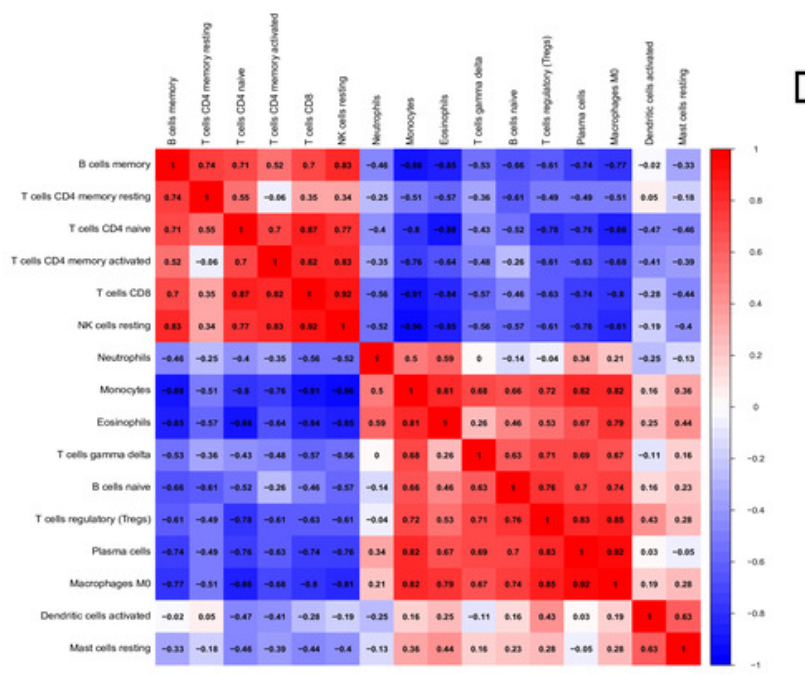

B

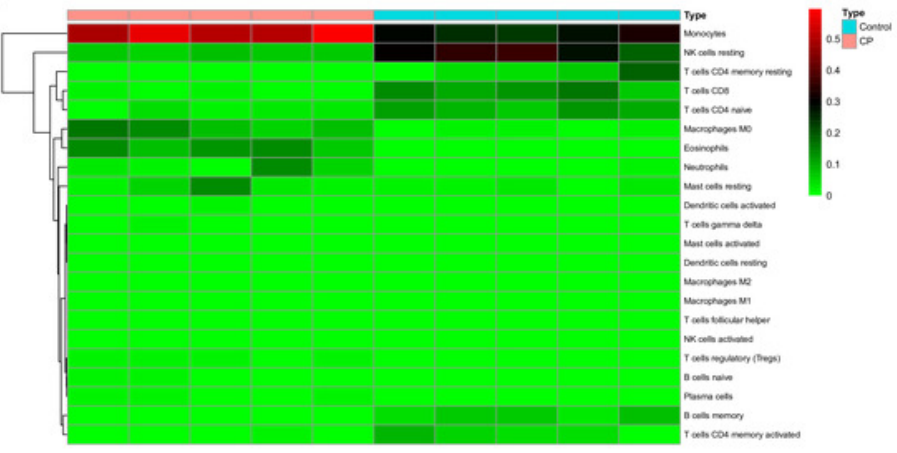

D

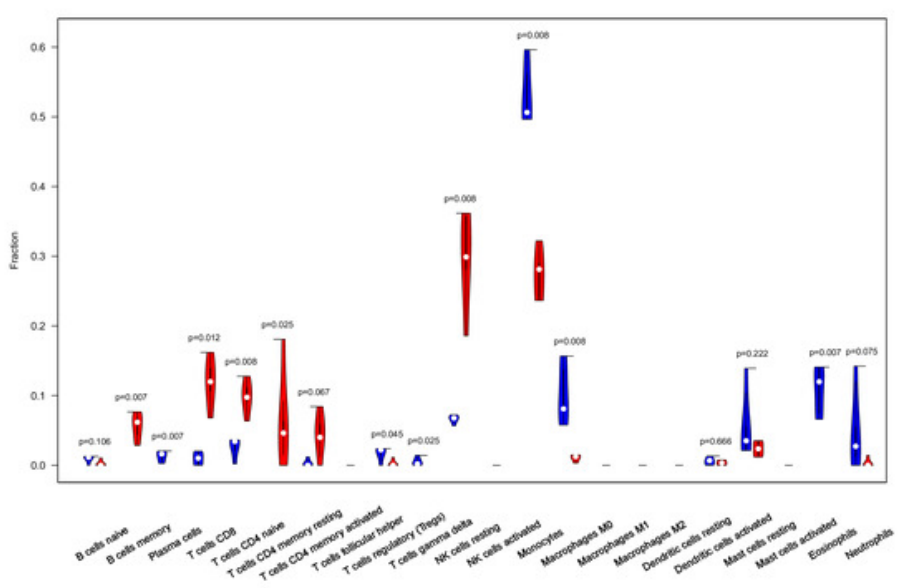


Figure 10

Relationships between the hub genes and immune cells in CML-CP-Normal
(A) CTSG. (B) MMP9. (C) PRTN3. (D) RETN. (E) RNASE2. (F) RNASE3.

A

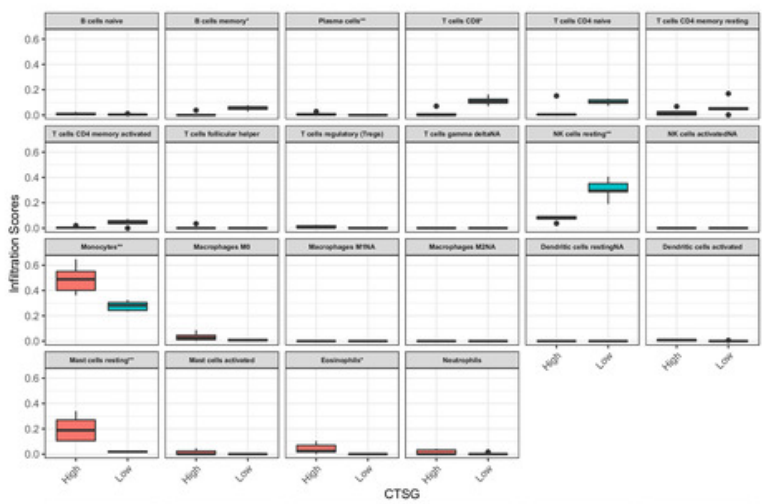

C

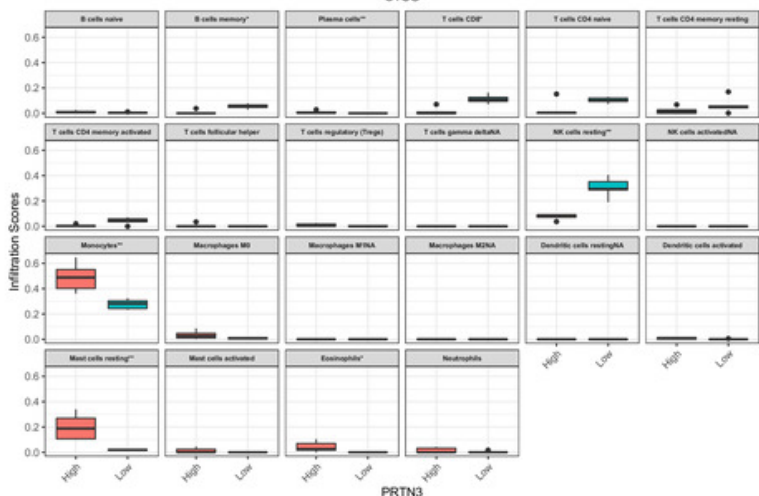

E

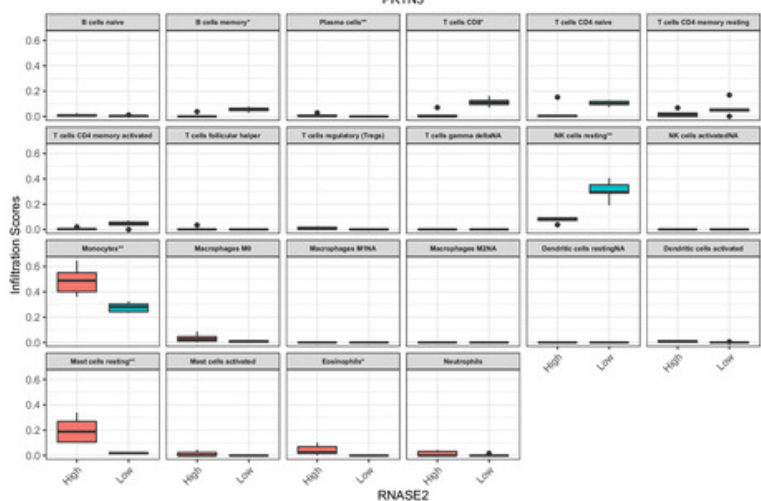

B

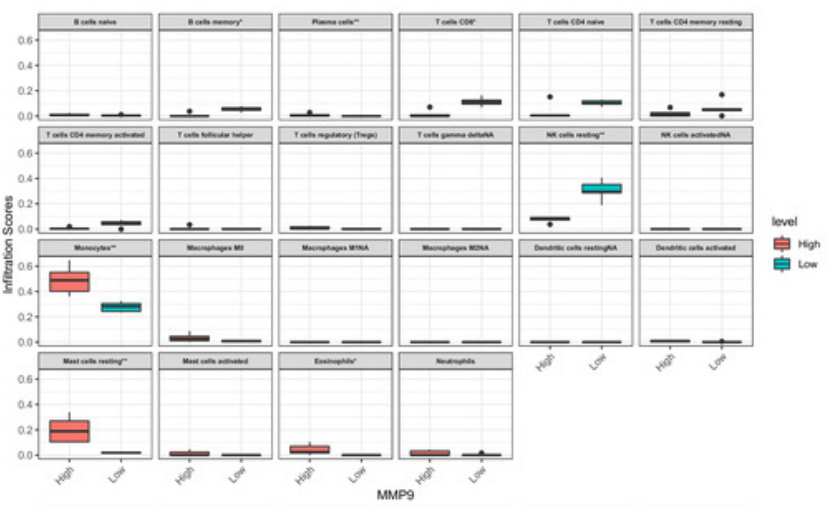

D

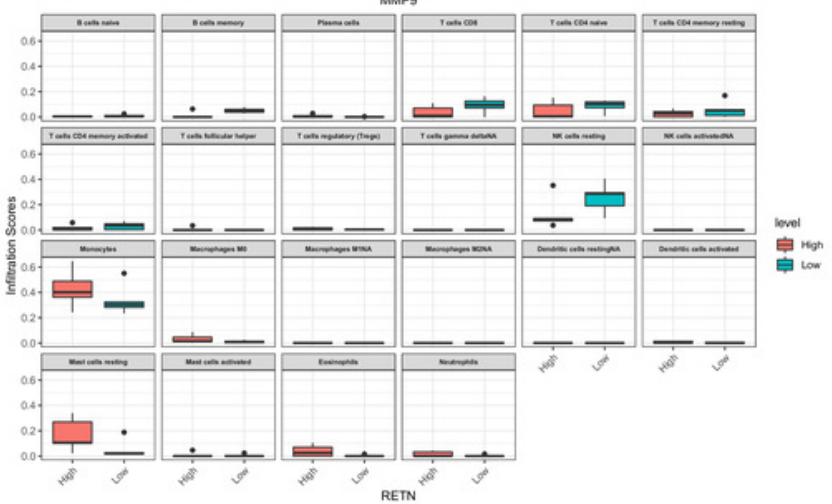

$\mathrm{F}$

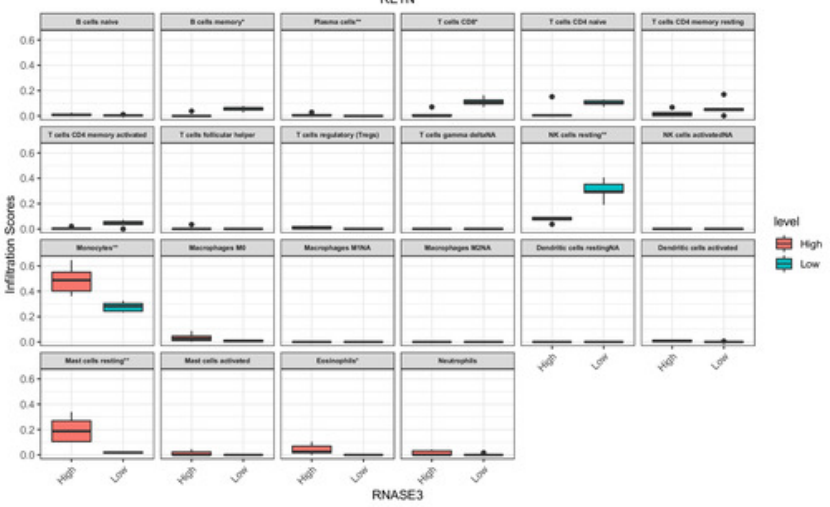


Figure 11

Relationships between the hub genes and immune cells in CML-BP-Normal
(A) CTSG. (B) MMP9. (C) PRTN3. (D) RETN. (E) RNASE2. (F) RNASE3.

A

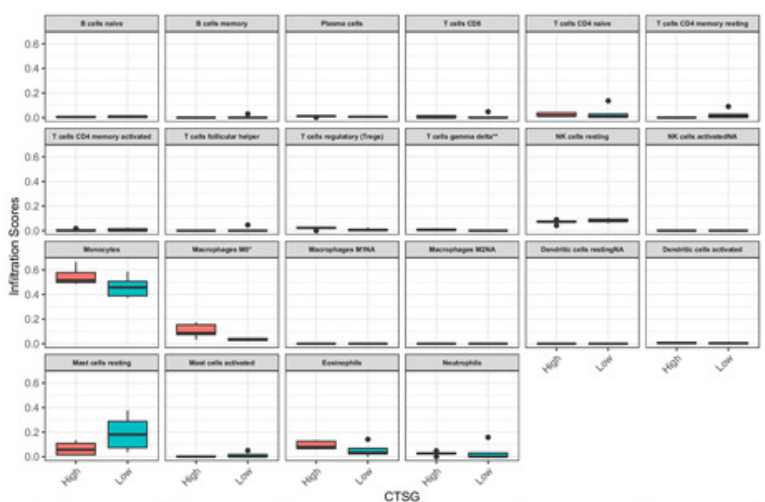

C

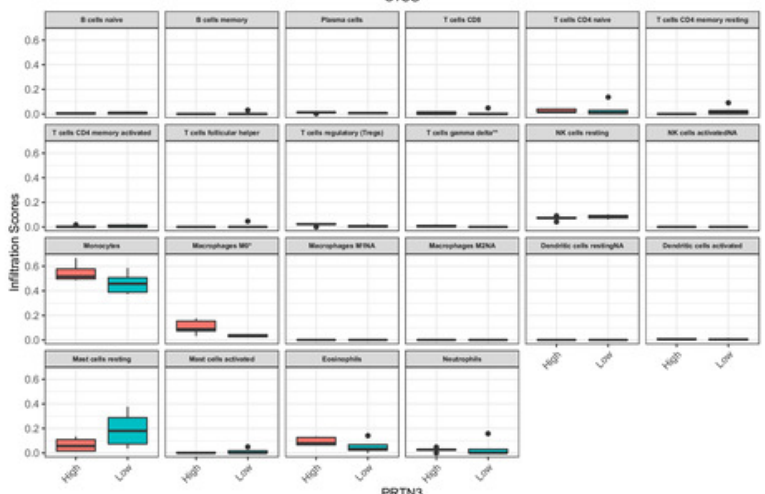

E

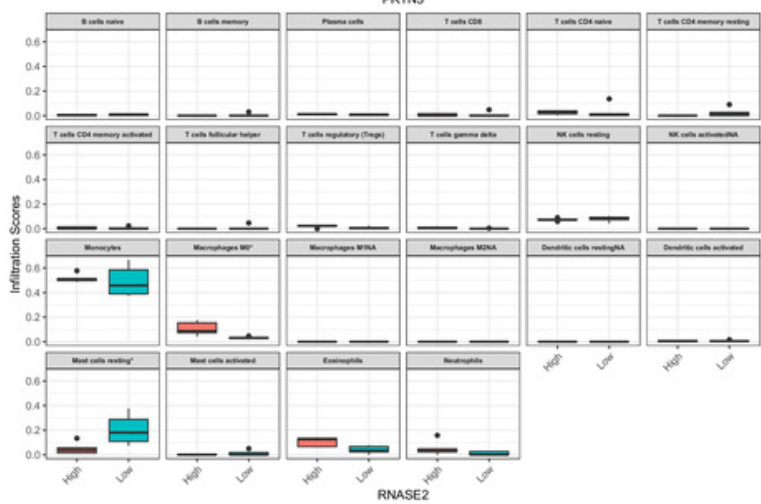

B

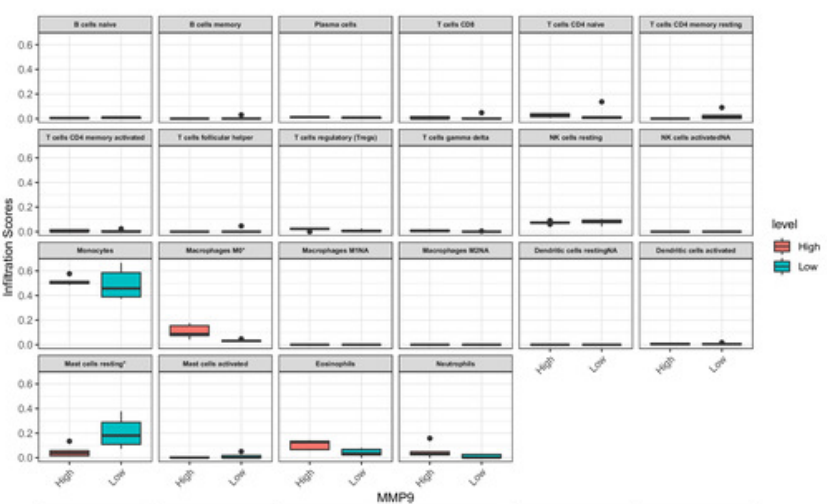

D

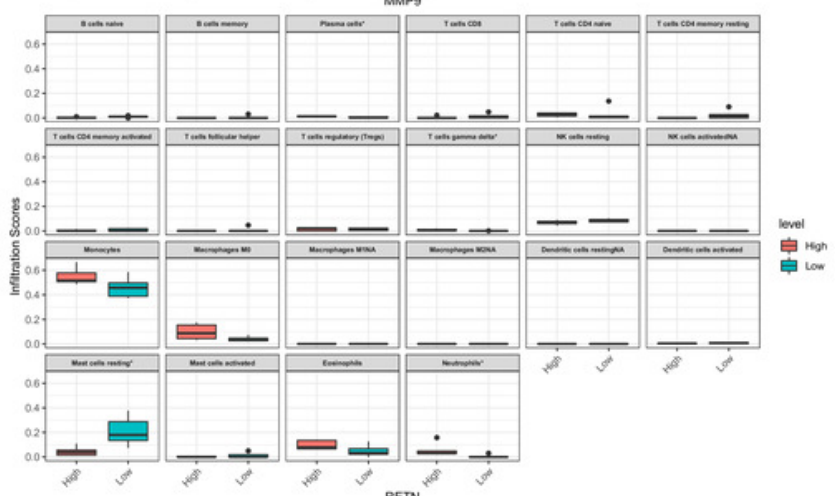

$\mathrm{F}$

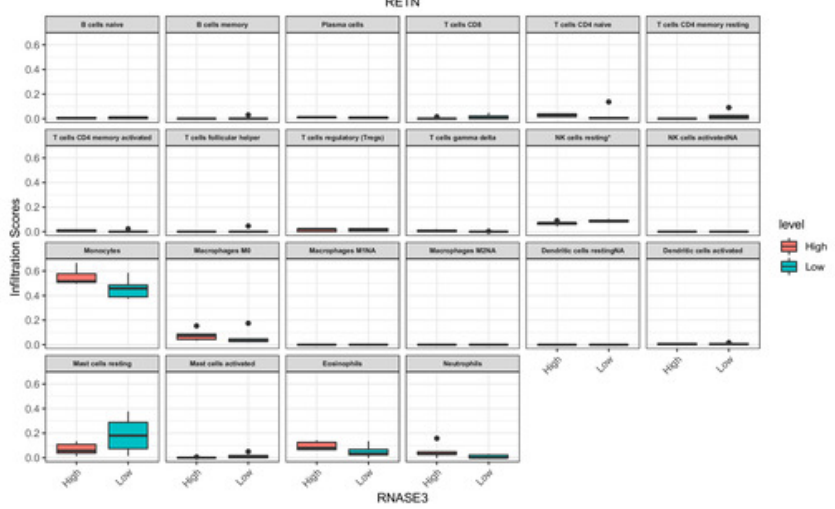


Figure 12

Relationships between the hub genes and immune cells in CML-CP-CML-BP
(A) CTSG. (B) MMP9. (C) PRTN3. (D) RETN. (E) RNASE2. (F) RNASE3.

A

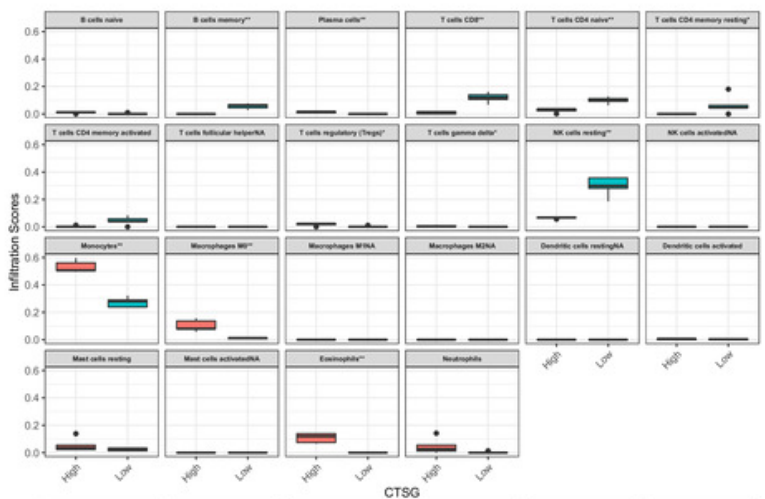

C

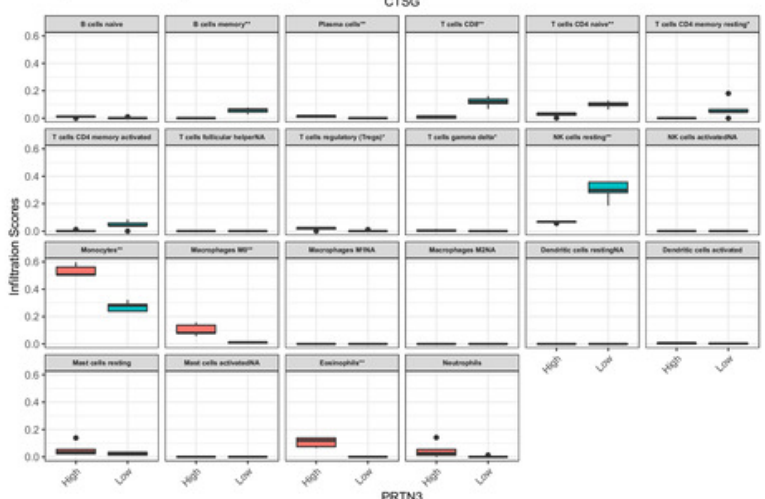

E

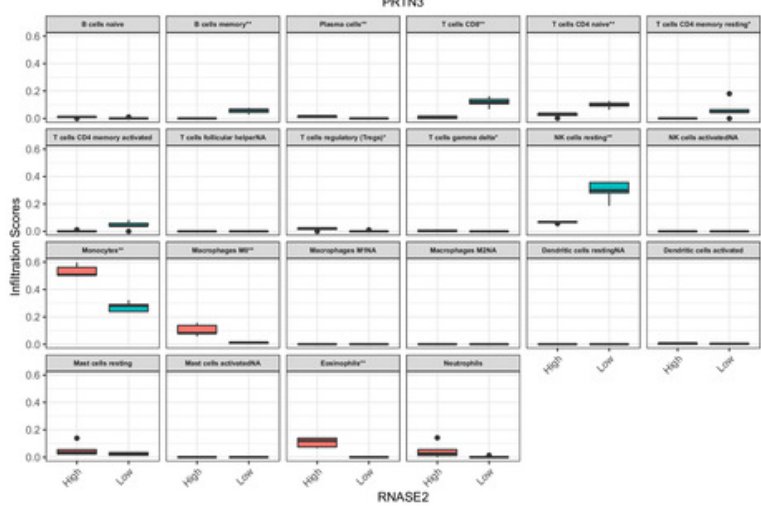

B

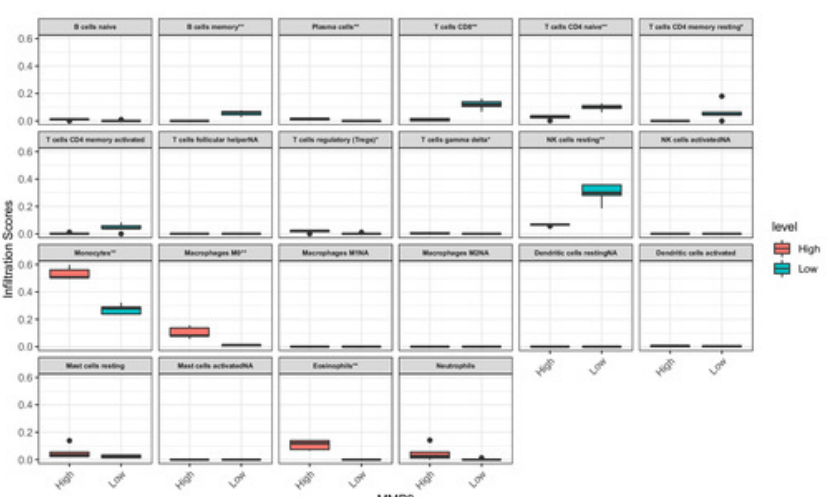

D

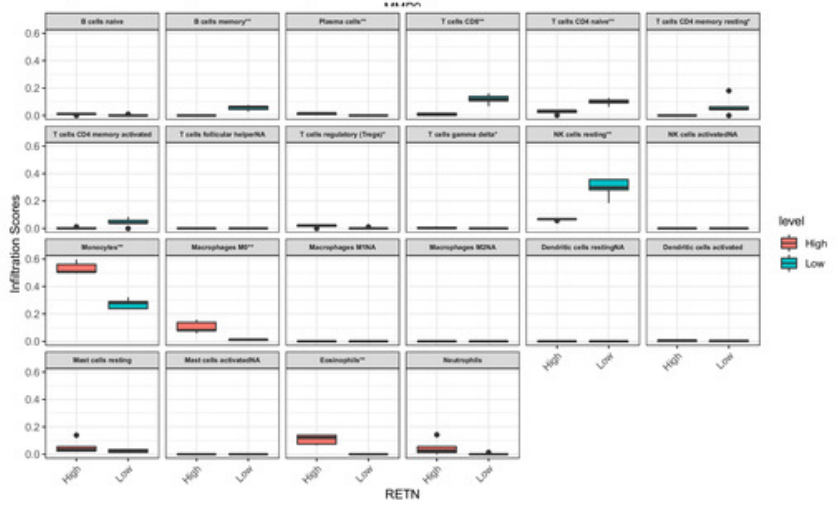

$\mathrm{F}$

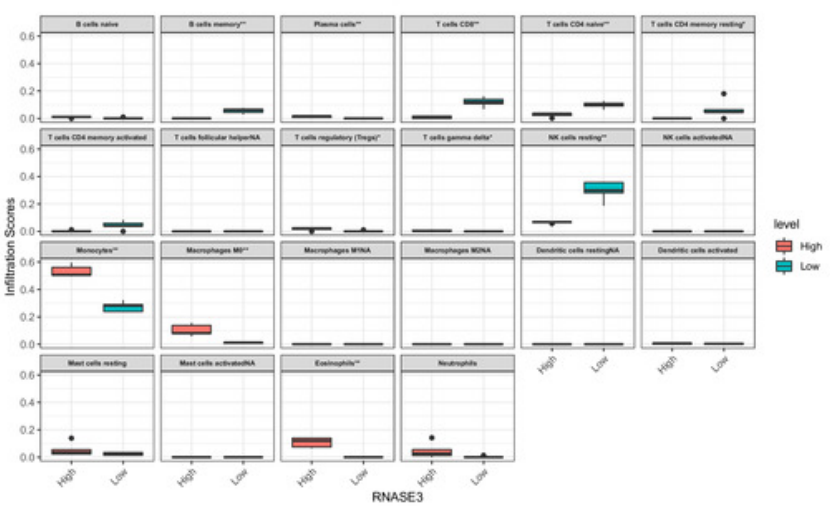


Figure 13

Related expression levels of hub genes

(A) CTSG. (B) MMP9. (C) PRTN3. (D) RETN. (E) RNASE2. (F) RNASE3. Significant differences were supposed at ${ }^{*} P<0.05 ;{ }^{* *} P<0.01 ;{ }^{* * *} P<0.001$.

A

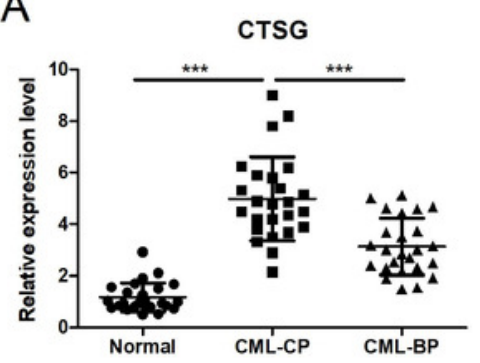

D

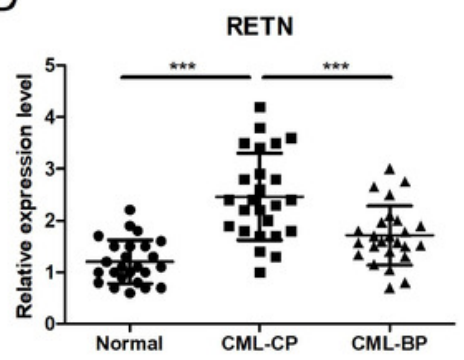

B

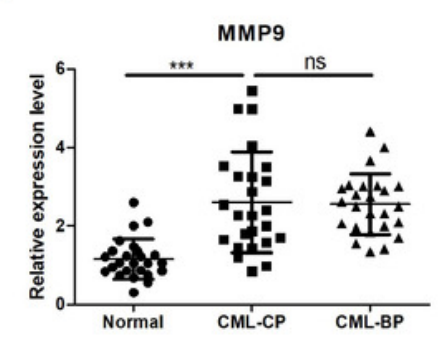

E

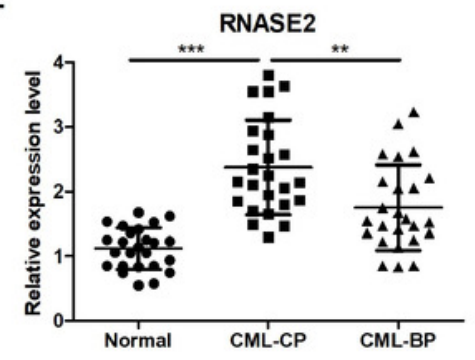

C

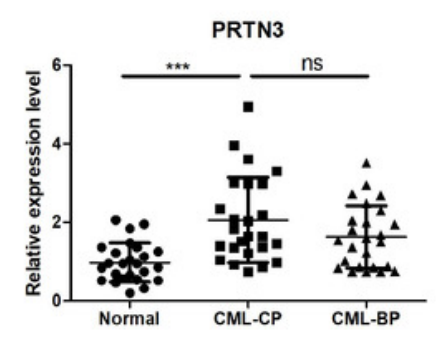

$\mathrm{F}$

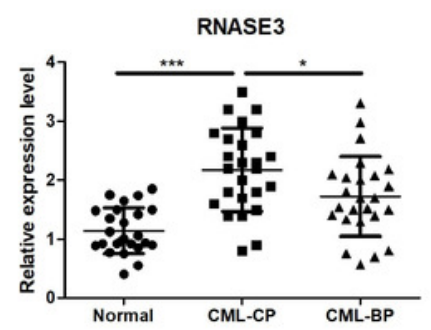

